San Jose State University

SJSU ScholarWorks

Master's Theses

Master's Theses and Graduate Research

1992

\title{
The adsorption of moisture on carbon films used as overcoats for rigid disk thin film magnetic recording media
}

Keith Goodson

San Jose State University

Follow this and additional works at: https://scholarworks.sjsu.edu/etd_theses

\section{Recommended Citation}

Goodson, Keith, "The adsorption of moisture on carbon films used as overcoats for rigid disk thin film magnetic recording media" (1992). Master's Theses. 460.

DOI: https://doi.org/10.31979/etd.yvks-ccg8

https://scholarworks.sjsu.edu/etd_theses/460

This Thesis is brought to you for free and open access by the Master's Theses and Graduate Research at SJSU ScholarWorks. It has been accepted for inclusion in Master's Theses by an authorized administrator of SJSU ScholarWorks. For more information, please contact scholarworks@sjsu.edu. 


\section{INFORMATION TO USERS}

This manuscript has been reproduced from the microfilm master. UMI films the text directly from the original or copy submitted. Thus, some thesis and dissertation copies are in typewriter face, while others may be from any type of computer printer.

The quality of this reproduction is dependent upon the quality of the copy submitted. Broken or indistinct print, colored or poor quality illustrations and photographs, print bleedthrough, substandard margins, and improper alignment can adversely affect reproduction.

In the unlikely event that the author did not send UMI a complete manuscript and there are missing pages, these will be noted. Also, if unauthorized copyright material had to be removed, a note will indicate the deletion.

Oversize materials (e.g., maps, drawings, charts) are reproduced by sectioning the original, beginning at the upper left-hand corner and continuing from left to right in equal sections with small overlaps. Each original is also photographed in one exposure and is included in reduced form at the back of the book.

Photographs included in the original manuscript have been reproduced xerographically in this copy. Higher quality $6 " \mathrm{~m}$ " 9 " black and white photographic prints are available for any photographs or illustrations appearing in this copy for an additional charge. Contact UMI directly to order.
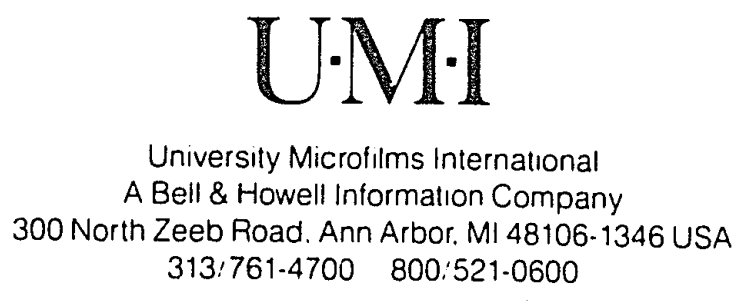
Order Number 1351031

The adsorption of moisture on carbon films used as overcoats for rigid disk thin film magnetic recording media

Goodson, Keith Samuel, M.S.

San Jose State University, 1992 

THE ADSORPTION OF MOISTURE ON CARBON FILMS USED AS OVERCOATS FOR RIGID DISK THIN FILM MAGNETIC RECORDING MEDIA

\author{
A Thesis \\ Presented to \\ The Faculty of the Department of Materials Engineering \\ San Jose State University \\ In Partial Fulfillment \\ of the Requirements for the Degree \\ Master of Science
}

By

Keith Goodson

December, 1992 
APPROVED FOR THE DEPARTMENT OF MATERIALS ENGINEERING

Gina Selvaduray

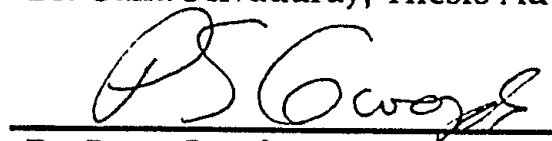

Dr. Peter Gwodz, Professor of Mat. Engr.

Uhlan S. Hodad

Allan Hadad, Conner Peripherals, Disk Division

APPROVED FOR THE UNIVERSITY

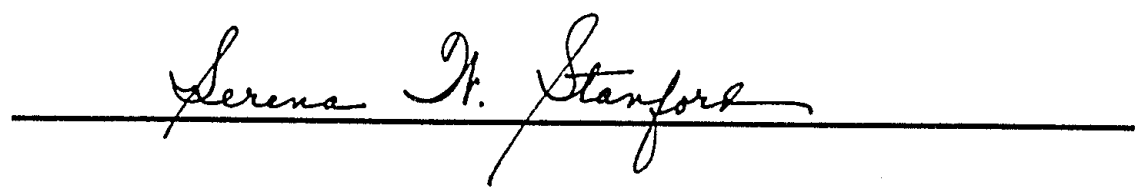




\section{ABSTRACT \\ The Adsorption of Moisture on Carbon Films Used as Overcoats for Rigid Disk Thin Film Magnetic Recording Media \\ by Keith Goodson}

The amount of moisture adsorbed on DC magnetron sputtered carbon was compared to the amount of moisture adsorbed on carbon deposited by plasma enhanced chemical vapor deposition. A technique was developed which used a quartz crystal microbalance and a simple environmental chamber. Duplicate carbon films were deposited at different thicknesses on quartz crystals and the adsorption and desorption of moisture was evaluated between 0 and $70 \%$ relative humidity (RH). A $50 \mathrm{~nm}$ thick DC magnetron carbon film had isothermal moisture adsorption measurements made at $24.5,29.5$ and $34.5^{\circ} \mathrm{C}$. The results showed that the amount of moisture adsorbed was proportional to the relative humidity and the thickness. The amount of moisture adsorbed was independent of temperature when measured as a function of RH. 


\section{ACKNOWLEDGMENTS}

The author wishes to thank Dr. Guna Selvaduray for his guidance and perseverance in seeing this project to its completion. Thanks are also due Dr. Peter

Gwodz for his careful comments. The author also wishes to thank Allan Hadad for his many technical and editorial comments during the writing of this thesis. A special thanks to Gayle Goodson for her support during the entire process and especially for help in preparing this manuscript. 


\section{TABLE OF CONTENTS}

\section{Page}

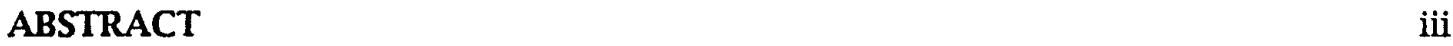

ACKNOWLEDGMENTS iv

LIST OF FIGURES vii

LIST OF TABLES ix

CHAPTER 1. Introduction 1

CHAPTER 2. Magnetic Rigid Disk Technology 3

2.1 Magnetic recording 3

2.2 Review of thin carbon films for magnetic rigid disks 9

$\begin{array}{ll}2.3 \text { Importance of moisture adsorption } & 11\end{array}$

$\begin{array}{ll}2.4 \text { Adsorption from the vapor phase } & 13\end{array}$

2.5 Quartz crystal microbalance theory and operation 20

CHAPTER 3. Research Objectives and Hypothesis 29

$\begin{array}{ll}\text { CHAPTER 4. Experimental Methodology } 30 & 30\end{array}$

$\begin{array}{ll}4.1 \text { Experimental overview } & 30\end{array}$

$\begin{array}{ll}4.2 \text { Adsorption apparatus } & 31\end{array}$

4.3 Evaluation of moisture adsorption on gold electrodes of quartz 37 crystals

4.4 Plasma enhanced chemical vapor deposition of carbon on gold 38 electrodes of quartz crystals

4.5 Deposition of DC magnetron sputtered carbon on gold electrodes of $\quad 40$ quartz crystals

4.6 Adsorption of moisture on carbon 42

4.7 Film characterization 43

CHAPTER 5. Results and Discussion $\quad 45$

$\begin{array}{ll}5.1 \text { Carbon analysis } & 45\end{array}$ 
5.2 Moisture adsorption on gold electrodes of quartz crystals 46

5.3 Moisture adsorption on DC magnetron sputtered carbon 50

5.4 Adsorption of moisture on DC magnetron sputtered carbon as a $\quad 60$ function of $\mathrm{RH}$ and temperature

5.5 Moisture adsorption on PECVD carbon $\quad 62$

5.6 Comparison of moisture adsorption on PECVD and DC magnetron $\quad 67$ sputtered carbon films

$\begin{array}{lr}\text { CHAPTER 6. Conclusions } & 69\end{array}$

CHAPTER 7. Recommendations for Future Research 70

CHAPTER 8. Endnotes $\quad 72$

$\begin{array}{ll}\text { APPENDIX } & 76\end{array}$ 


\section{LIST OF FIGURES}

Figure

Page

1 Mlustration of the magnetic recording process 4

2 Effect of head medium separation on signal output 6

3 Typical magnetic thin film cross section $\quad 7$

4 Typical magnetic recording element and slider body 8

5 Coefficient of kinetic friction versus relative humidity (RH) 12

6 Schematic diagram of BET adsorption isotherms 16

7 Thickness -shear mode of vibration 22

8 AT -cut quartz crystal $\quad 23$

9 Frequency change vs. temperature for an AT crystal cut at 35 $15^{\prime} \quad 26$

10 Schematic diagram of adsorption apparatus 32

11 Front view photograph of the adsorption apparatus 33

12 Rear view photograph of the adsorption apparatus 34

13 Leybold-Inficon standard crystal sensor 35

14 PECVD system $\quad 39$

15 Schematic drawing of a DC magnetron sputtering system 41

16 Adsorption of moisture on gold electrodes of quartz crystals 47

17 Adsorption of moisture on DC magnetron sputtered carbon $\left(24.5^{\circ} \mathrm{C}\right)$

18 Three dimensional AFM scan of $25 \mathrm{~nm}$ thick DC magnetron carbon 55 film

19 Three dimensional line scan of $100 \mathrm{~nm}$ thick DC magnetron carbon 56 film

20 AFM line scan of $25 \mathrm{~nm}$ thick DC magnetron deposited carbon 57

21 AFM line scan of $100 \mathrm{~nm}$ thick DC magnetron deposited carbon 58 
Figure

Page

22 Adsorption of moisture on $50 \mathrm{~nm}$ thick DC magnetron sputtered carbon as a function of temperature and $\mathrm{RH}$

23 Moisture adsorption on PECVD carbon as a function of $\mathrm{RH}\left(24.5^{\circ} \mathrm{C}\right)$

24 AFM line scan of $50 \mathrm{~nm}$ thick PECVD carbon

25 AFM line scan of $100 \mathrm{~nm}$ thick PECVD carbon

66

26 Adsorption of moisture on DC magnetron sputtered carbon and on

68 PECVD carbon as a function of relative humidity $\left(24.5^{\circ} \mathrm{C}\right)$ 


\section{LIST OF TABLES}

\section{Table}

1 Typical properties of sputtered and PECVD carbon 11

2 The piezoelectric constant of various materials 21

3 Carbon analysis: PECVD versus DC magnetron sputtered 45

4 Carbon properties: PECVD versus DC magnetron sputtered 46

5 Student t-test for significant differences in mass of adsorbed moisture 52 at $70 \%$ relative humidity versus sputtered carbon thickness

6 The relative surface area of DC magnetron sputtered carbon as a 53 function of thickness

7 Student t-test for significant differences in amount of moisture 62 adsorption at $70 \%$ relative humidity versus thickness of PECVD carbon

8 The relative surface area of PECVD carbon as a function of thickness 


\section{CHAPTER 1}

Introduction

The development, mass production, and sale of personal computers has continued to fuel the rapid growth of magnetic rigid disk drives. These devices which are used as permanent memory for computers generated world wide revenues of $\$ 20.4$ billion dollars in 1988 and by 1990 revenues increased to almost $\$ 27$ billion dollars.(1) In 1992 they are expected to exceed $\$ 32$ billion.(2)

In the U.S.A. alone there are over 30 manufacturers of rigid disk drives. The rapid development of the technology in other nations (primarily Japan) has increased the world wide number of disk drive manufacturing companies to almost 60 .

The manufacturing of rigid disk drives has also created a number of supporting industries. Among these are the production of magnetic disks, magnetic heads, disk substrate materials, and related magnetic head, magnetic disk, and disk drive test equipment. These industries have developed several intensive areas of research. One of the most important is the study of friction and wear properties of the thin carbon films used as overcoats for magnetic disks.

This study was undertaken to investigate the capability of carbon thin films to adsorb moisture. In order to accomplish this, a quartz crystal microbalance technique was developed. This allowed the comparison of moisture adsorbed on carbon thin films deposited by sputtering versus those deposited by plasma enhanced chemical vapor deposition.

Chapter 2 reviews magnetic rigid disk technology, the importance of carbon thin films on magnetic rigid disks, the importance of moisture adsorption and theoretical aspects of adsorption. The chapter concludes with a discussion of the quartz crystal microbalance theory and operation. The research objectives are stated in Chapter 3 , 
followed by experimental details in Chapter 4 . Chapter 5 summarizes and discusses the results obtained in this investigation. Conclusions, recommendations for future research, and references are presented in Chapters 6, 7 and 8, respectively. 


\section{CHAPTER 2}

\section{Magnetic Rigid Disk Technology}

\subsection{Magnetic recording}

The rotating rigid disk for digital data storage applications was introduced in 1957.(3) High reliability and magnetic data transfer rates were provided by a rapidly rotating (typically 3600 RPM) rigid disk over which a magnetic read-write head moved in and out. Data was recorded as magnetic patterns written in circles around the hub of the disk. Each of the concentric circles made up a track and each track was divided into a number of equal segments called sectors. Once the magnetic head was in position it would wait for the correct sector to rotate beneath it and then read or write data. Many refinements have occurred since 1957 but modern disk drives still operate under the same basic principle.

In order to preserve the integrity of this head disk interface (HDI), flying head technology was developed.(4) In this technique, the head is supported by a thin air bearing during normal rapid disk rotation (typically 3600 RPM) and touches the disk surface only during start and stop operations. When the disk starts rotating the recording head drags on the disk surface until a critical disk speed is reached at which time the air pressure developed under the head exceeds the force pushing the head towards the disk. At this point a typical 0.1-0.25 $\mu \mathrm{m}$ (4 to $10 \mu \mathrm{in})$ head-to-disk separation develops. The reverse happens in the stop process when the disk slows down. When metallic thin film disks replaced oxide coated disks, it was found that a thin $(20-100 \mathrm{~nm})$ sputtered carbon film was necessary to protect the head and disk during these start and stop operations. ${ }^{(5)}$ Without a wear resistant coating, wear between the head and disk could lead to rapid degradation in the readback signal amplitude, or even catastrophic failure, due to destruction of the head, or disk, or both. 
Several texts ${ }^{(6,7)}$ have covered the principles of magnetic recording in detail. Greatly simplified, a magnetic recording system consists of a permanent magnet in which small areas are permanently magnetized in a specific direction by the application of an external magnetic field. Figure 1 shows the medium in the form of a magnetic layer supported by a non-magnetic substrate. The transducer or recording head is a ring-shaped electromagnet with a gap at the front facing the medium. When a current flows through the conductive wires (coil) wrapping the recording head a magnetic field appears at the head gap.

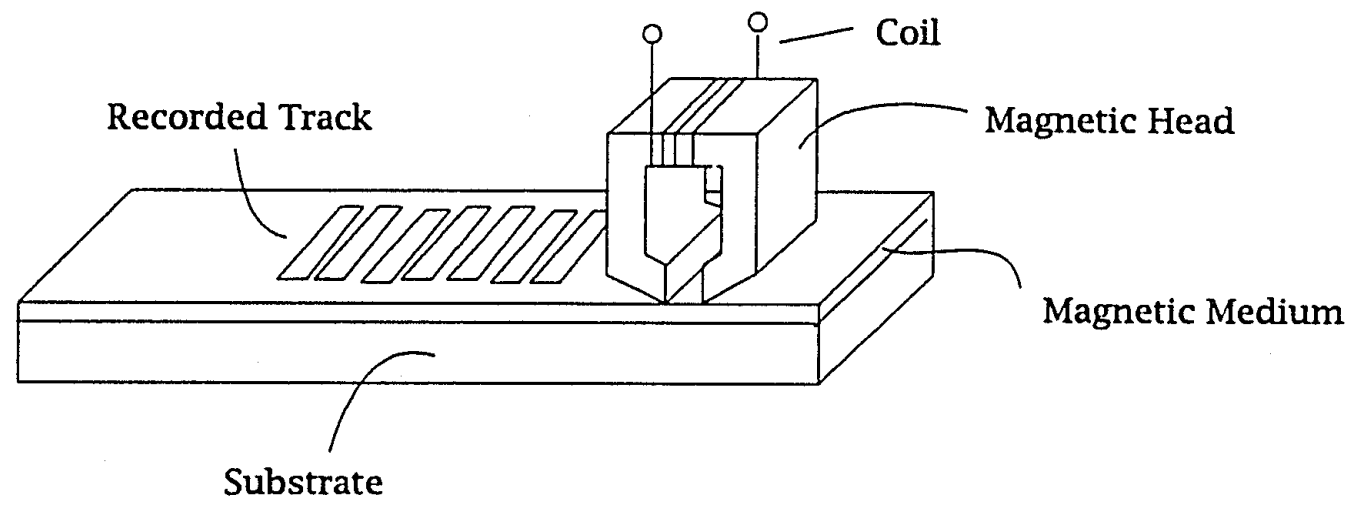

Figure 1. Mlustration of the magnetic recording process

This field will leave small areas of remanent magnetization on the medium parallel to the direction of the applied field. The areas of local magnetization will remain on the medium indefinitely unless the magnetic material is damaged, reduced to a non magnetic state, for example by corrosion, or magnetically erased. This recording process is known as longitudinal recording. While there are competing technologies such as vertical recording, they will not be described here since they are not a major commercial process at this time. 
When the previously recorded medium is passed close to the gap in the magnetic transducer, a voltage is induced in the coil. This voltage is not an exact replica of the recording signal, but by appropriate electronic manipulation the recording signal can be defined. The empirical relationship, developed by Faraday, which relates the voltage induced in the coil $(e)$ to the velocity of relative motion between the recorded medium and the head $(V)$, the number of turns on the head $(N)$, and the change in flux $(\phi)$ with respect to distance $(x)$ in the recorded medium is given by Equation 1 .

$$
e=-N V(\mathrm{~d} \phi / \mathrm{d} x)
$$

Another important magnetic recording relationship is the output voltage of the readback signal versus the spacing between the magnetic head and magnetic medium. Figure 2 illustrates ${ }^{(8)}$ the rapid increase in signal output with decreasing distance between the head and disk. As can be seen in Figure 2, one of the techniques of increasing information storage density is to fly the head closer to the disk and ultimately even touch or slide along the disk surface. (9) This, however, may increase the risk of catastrophic failure since the debris generated with wear is now closer to the flying head. Also, as the head and disk separation decreases, incidental contacts between the head and disk increase. As the density of the recorded bits in Figure 1 increases (bits/inch) the output signal will decrease. This phenomenon is largely an effect of magnetic interference (self erasure) between adjacent cells. This is another reason the magnetic head will have to be brought closer to the magnetic medium and ultimately contact the disk surface. In pushing towards higher storage density a key factor is the interface between the magnetic recording head and the thin film disk 
surface. The need for understanding of the head-disk interface tribology will continue to increase in importance as flying heights are reduced.

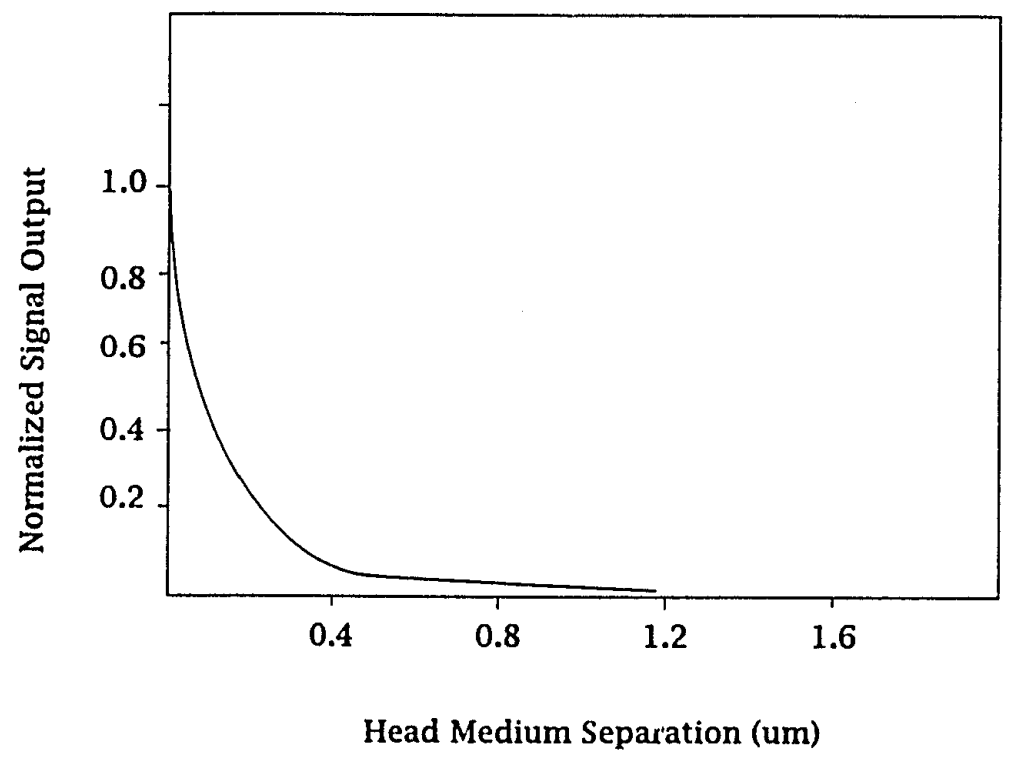

Figure 2. Effect of head medium separation on signal output

Figure 3 shows a cross-section of a typical thin film rigid disk. The substrate is an aluminum disk, typically a 5586T2 alloy. A layer of non-magnetic nickelphosphorous is plated on the surface. The nickel-phosphorous layer provides a hard surface which can be readily polished to the fine finish required. The magnetic layer itself generally consists of a cobalt alloy, although other materials such as metal oxides have also been investigated. The magnetic layer can be applied by plating or sputtering. If the plating process is used, cobalt alloy is applied directly to the polished nickelphosphorous. If the magnetic layer is applied by the sputtering process, a chromium 
intermediate layer must be sputtered to provide the correct crystalline orientation of the subsequently deposited cobalt layer.

Finally, a thin layer of carbon is deposited to provide lubrication, and improve wear and corrosion properties. While not shown in Figure 3, a thin lubricant film (1.5-4.0 $\mathrm{nm})$, typically a perfluoropolyether material, is applied on top of the carbon overcoat.

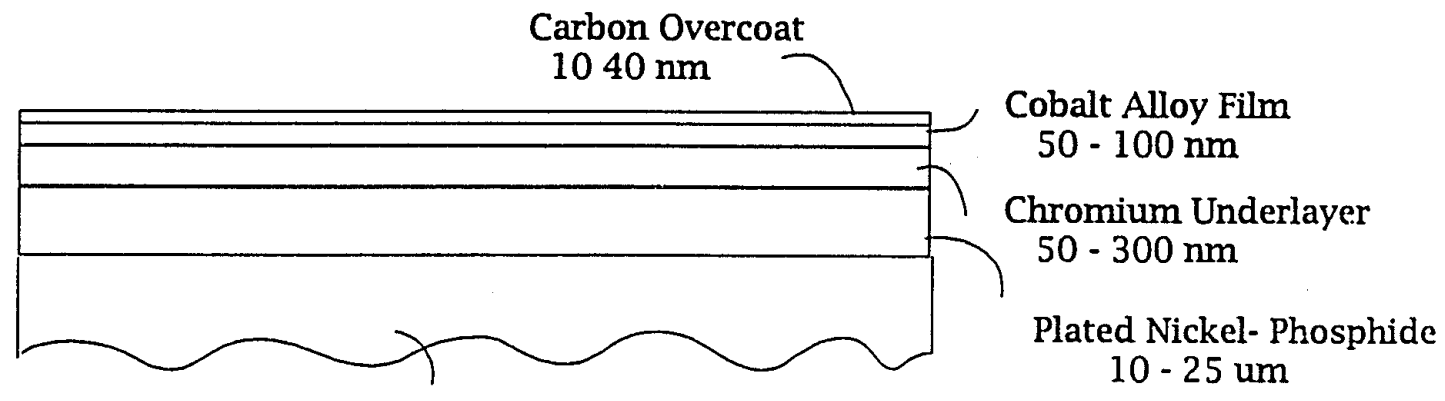

Al-Mg alloy substrate

Figure 3. Typical magnetic thin film cross section

A schematic diagram of a magnetic rigid disk head is shown in Figure 4 . This head not only contains the magnetic read-write element shown in Figure 1 but also a much larger structure commonly called the slider. Several of the common ceramic materials used in the construction of the slider are also shown in Figure 4 . It is the slider surface which dominates the mechanical behavior of the head. This is due to its larger volume and contact area. The slider configuration, and materials of construction, along with the surface of the disk to a large extent determines the tribology of the head disk interface.

Several technological aspects of magnetic rigid disks have been reviewed in this introduction. Emphasis has been placed on the need to lower the flying height of the 


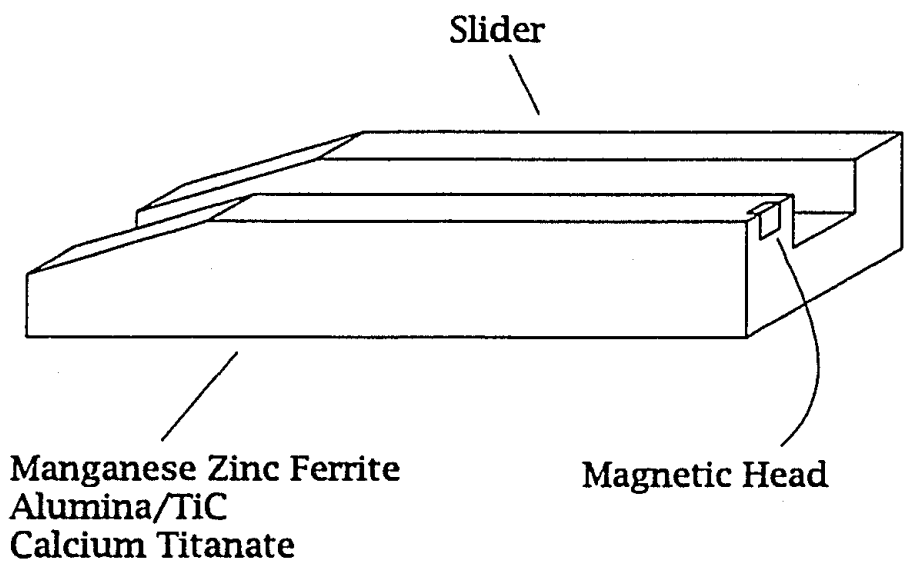

Figure 4. Typical magnetic recording element and slider body

magnetic head and the challenges this poses for reliable operation of the disk drive. The ultimate goal of contact recording will require new understanding of the friction and wear properties of the head disk interface. The increase in magnetic information density will also put new demands on understanding failure mechanisms such as corrosion, which can degrade the magnetic properties of the rigid disk.

Knowledge of the relationship between the partial pressure of moisture in the air and the amount of moisture adsorbed on the surface of a magnetic disk will make a fundamental contribution in two areas of magnetic recording. The surface moisture is known to have an effect on the mechanical interface between the magnetic recording media and the magnetic recording head.(10) The adsorbed moisture is also known to participate in the electrochemical corrosion ${ }^{(11)}$ of the cobalt alloy magnetic disk. If the amount of moisture adsorbed is affected by the type of carbon thin film both the mechanical interface and disk corrosion could be improved by proper control of the carbon film. 


\subsection{Review of thin carbon films for magnetic rigid disks}

Elemental carbon occurs widely in both crystalline and amorphous elemental forms. Diamond and graphite are the two crystalline allotropes of carbon. These two allotropes differ in their physical and chemical properties because of differences in the arrangement and bonding of the atoms. ${ }^{(12)}$ The diamond crystal structure is face centered cubic with interatomic distances of $0.154 \mathrm{~nm}$. Each atom is covalently bonded to four other carbon atoms via $\mathrm{sp}^{3}$ tetragonal bonds. The density is $3.51 \mathrm{~g} / \mathrm{cm}^{3}$. The structure of graphite is described as layers of carbon atoms with strong $\mathrm{sp}^{2}$ trigonal bonds. The separation of the layers is $0.335 \mathrm{~nm}$ and the density is $2.22 \mathrm{gm} / \mathrm{cm}^{3}$. A fourth bond in the outer shell forms a weak bond of the van der Waals type between the planes and is responsible for good electrical conductivity, lubricity, lower density, and softness.

The many forms of so called amorphous carbon, such as charcoal, soot, and lampblack, are all microcrystalline forms of graphite. The physical properties of such materials are largely determined by the nature and magnitude of their surface areas and tend to vary depending on the method of preparation.

In a study of the tribology of the head disk interface Leu ${ }^{(13)}$ referenced 26 papers on carbon thin films that had been published in the past 10 years. He also noted that the first publications in this field date to 1956. Promising applications for carbon thin films spread across the metallurgical, surface finishing, semiconductor, and optical industries. They are also under study or have found applications in IR optical elements, Si-solar cells and as tribological coatings for improved wear resistance of metal parts. Leu noted that carbon films have been grown by many different techniques including: evaporation, sputtering, ion beams, and plasma enhanced chemical vapor 
deposition (PECVD). In a 1987 review Bogy and Tsai(14) cited 176 references covering the preparation and characterization of thin carbon films.

The decomposition of a hydrocarbon gas in a glow discharge and the subsequent deposition on a substrate is called plasma enhanced chemical vapor deposition (PECVD). The harder, less conductive, carbon films are often referred to in the literature as "diamond-like-carbon" (DLC) films but this term has also been applied to any carbon thin film deposited by physical or chemical techniques. In the PECVD technique hydrogen can also be included in the film. When hydrogen is included in the film it is sometimes referred to in the literature as a sub-class of DLC, amorphous hydrogenated carbon $(\mathrm{a}-\mathrm{C}: \mathrm{H})$. The macroscopic properties of DLC a-C:H are determined by the hydrogen content and the medium range structure of the a-C:H network. (15)

Plasma enhanced chemical vapor deposition is conceptually similar to ion beam deposition. The glow discharge acts as an ion source and the positive hydrocarbon ions are accelerated across the ion sheath towards the cathode mounted substrate. The energy with which the ion strikes the cathode is controlled by the bias voltage. The hydrocarbon pressure is fixed at a few $\mathrm{Pa}$.

The majority of magnetic disks produced today are over-coated with sputtered carbon. In sputtering, a plasma is generated in an argon filled vacuum chamber by connecting a DC or RF voltage source to a pyrolytic graphitic target. The carbon films are deposited onto the substrate from a carbon species obtained by argon ion bombardment of the graphitic target.

Properties of carbon films cannot be easily summarized for each process since large variations within each process can substantially affect the film. In general it is recognized that the films containing more $\mathrm{sp}^{3}$ bond characteristics have higher hardness, higher electrical resistivity and are more chemically inert. This is illustrated 
in Figure 1 where the properties of PECVD carbon and sputtered carbon are compared. In general sputtering has produced films with lower hardness and higher conductivity thus indicating decreased tetrahedral bonding.

Table 1 - Typical properties of sputtered and PECVD carbon ${ }^{(16)}$

\begin{tabular}{llcc}
\hline Process & $\begin{array}{l}\text { Density } \\
\mathrm{g} / \mathrm{cc}\end{array}$ & $\begin{array}{c}\text { Hardness } \\
\mathrm{kg} / \mathrm{mm}^{2}\end{array}$ & $\begin{array}{c}\text { Resistivity } \\
\text { Ohm-cm }\end{array}$ \\
\hline Sputter & $1.9-2.2$ & $1000-2000$ & $10^{4}$ \\
PECVD & $1.9-2.2$ & $1600-2600$ & $10^{11}$ \\
\hline
\end{tabular}

\subsection{Importance of moisture adsorption}

The coefficient of friction for DLC films sliding on steel has been reported to be strongly dependent on the relative humidity (RH) of the test atmosphere.(17) Coefficient of friction values of 0.01-0.02 were obtained at values of $\mathrm{RH}$ up to 1\%. Above 1\% $\mathrm{RH}$, the coefficient of friction increases rapidly and reaches about 0.2 at a $\mathrm{RH}$ of nearly 100\%. The relationship between coefficient of kinetic friction and sliding distance as a function of relative humidity for a thin film metal disk is shown in Figure 5. This work reported by Timsit and Stratford ${ }^{(18)}$ was on a carbon coated disk which was overcoated with a perfluoropolyether lubricant. The work shows that the kinetic friction increases as the relative humidity increases.

The tribology of the head disk interface is certainly very complicated and involves many factors. It is well known that the coefficient of friction has a significant effect on the wear rate.(19) The influence of relative humidity on frictional properties 


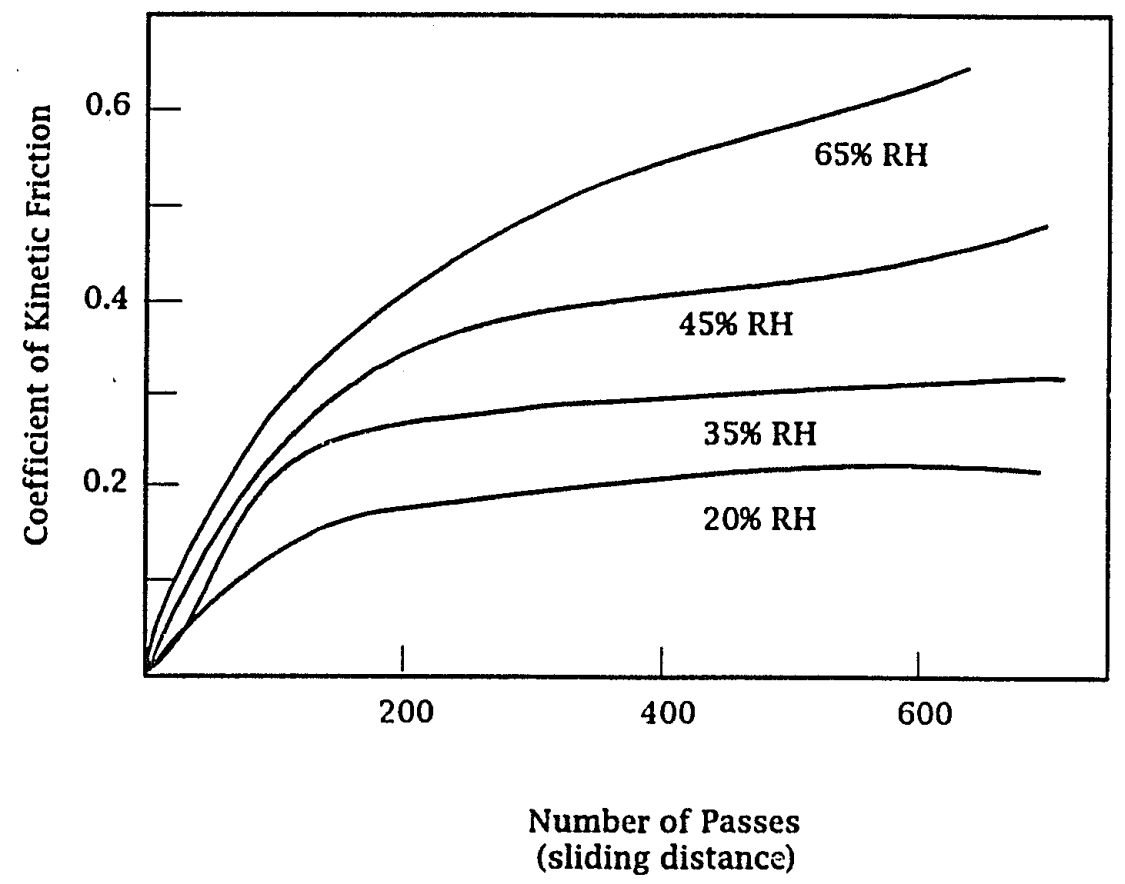

Figure 5. Coefficient of kinetic friction versus relative humidity (RH)

indicates that the adsorption of moisture on the carbon surface may be the effect that is actually being observed when researchers report changes in friction properties as a function of relative humidity. Understanding the amount of moisture adsorbed and also the variation of amount adsorbed with type of carbon will help in understanding and designing carbon overcoats for future recording applications.

Moisture adsorption may not only be important for friction and wear but may also play a part in the corrosion of the cobalt alloy on thin film disks. Current high performance disk drives are recording at linear densities up to 1,530 flux changes per millimeter (39,000 flux changes per inch, $\mathrm{FCI})$ and track densities of up to 79 tracks per millimeter (2500 tracks per inch, TPI). Within the next two years these densities are expected to reach 80,000 FCI at 2500 TPI with emerging technologies such as contact vertical recording.(20) A calculation for an $80,000 \mathrm{FCI}, 2500 \mathrm{TPI}$, disk shows that the 
corrosion of less than $3 \mu \mathrm{m}^{2}$ of cobalt at a depth of 30 to $60 \mathrm{~nm}$ can result in the loss of 1 bit of information. Substantially smaller corrosion volumes will produce data loss in future systems.

Concern regarding corrosion reliability has lead to numerous papers and reviews. ${ }^{(21,22)}$ Corrosion studies on cobalt thin film magnetic alloys include Co-P, $(23,24)$ Co-Ni-P, ${ }^{(25)} \mathrm{Co}-\mathrm{Pt} / \mathrm{Co}-\mathrm{Ni}-\mathrm{Pt}{ }_{i}^{(26)} \mathrm{Co}^{-\mathrm{Cr}^{(27,28)}}$ and $\mathrm{Co}-\mathrm{Ni}-\mathrm{M}^{(29)}$ where $\mathrm{M}$ is $\mathrm{Zr}, \mathrm{Cr}, \mathrm{Mo}$, or Pt. In general, the above studies have verified the electrochemical nature of the corrosion of the cobalt alloy and have shown that corrosion characteristics are dependent on alloy composition, processing conditions, overcoat, and atmospheric contaminants.

In the electrochemical mechanism, water adsorbs on the already hydroxylated cobalt surface in macroscopic in the form of stabilized droplets. Within these droplets corrosion occurs as localized oxidation-reduction reactions. While the electrochemistry of the aqueous micro regions is complex, it is clear that adsorbed water, ionic contaminants and surface morphology provide the basis for the electrochemical attack. ${ }^{(30)}$ Recent studies ${ }^{(31)}$ have proposed that galvanic action between the carbon surface and the cobalt alloy provides a corrosion mechanism in which the adsorbed water acts as the ionic conduit.

Adsorbed water can be a factor in the tribology of the head disk interface and the corrosion of the cobalt alloy disk. An understanding of both the amount of moisture adsorbed as a function of relative humidity and the variation of adsorption with carbon overcoat properties will help to solve existing problems leading to improved magnetic recording reliability.

\subsection{Adsorption from the vapor phase}

When a gas or vapor is brought into contact with a clean solid surface some of it will become attached to the surface in the form of an adsorbed layer. The solid is 
generally referred to as the adsorbent and the gas or vapor as the adsorbate or adsorptive. The adsorption is brought about by the forces acting between the solid and the molecules of the gas. These forces are of two main kinds, physical and chemical. The physical forces are the same in nature as van der Waals forces which bring about the transition from the vapor to the liquid state. Chemical adsorption is distinguishable qualitatively from physical adsorption in that the energy of adsorption is large enough to suggest that full chemical bonding has occurred.

Adsorption reduces the imbalance of attractive forces which exist at a surface and therefore reduces the surface free energy. When adsorption takes place, the molecules become immobilized or they are restricted to two dimensional motion. Adsorption processes are therefore accompanied by a decrease in entropy. Since the adsorption also involves a decrease in free energy, from the thermodynamic relationship

$$
\Delta G=\Delta H-T \Delta S
$$

it is evident that $\Delta H$ must always be negative, i.e. all adsorption processes are exothermic.

The forces which bring about adsorption always include London dispersion forces which are attractive, together with short range repulsive forces. Dispersion forces were first characterized by London(32) and are forces which arise from the rapid fluctuation in electron density within each atom. This induces an electrical moment in a near neighbor atom which leads to attraction between the two atoms. In addition there will be electrostatic (coulombic) forces if either the solid or the gas is polar in nature.

The development of London dispersion equations have lead to the understanding of adsorption processes on some very simple surfaces with carefully 
characterized gases. However, their development has not reached the state where it would be possible to calculate the detailed course of an adsorption isotherm from known and independently determined parameters of the gas and solid. (33)

Three phenomena may be involved in physical adsorption:

1. Monomolecular adsorption

2. Multimolecular adsorption

3. Condensation in pores or capillaries.

Frequently the adsorption phenomena overlap and the interpretation of adsorption studies can be complicated.

Brunauer, Emmett, Deming, and Teller, ${ }^{(34)}$ proposed that adsorption isotherms could be grouped into five classes. These have become commonly known as the BET, or Brunauer adsorption classification. The main characteristic of these types of adsorption isotherms are shown in Figure 6.

Type I isotherms show a fairly large rapid rise in the amount of adsorption with increasing vapor or gas concentration up to a limiting value. They are obtained when adsorption is restricted to a monolayer. Chemisorption isotherms may have this shape. Solids containing a very fine pore structure may also have this shape.

Type II isotherms are explained by Brunauer as resulting from multilayer physical adsorption on non-porous solids. Physical adsorption on microporous solids can also result in Type II isotherms. In this case, point B represents both monolayer adsorption on the surface as a whole and condensation in the fine pores. The remainder of the curve represents multilayer adsorption for non-porous solids.

Type III and Type V isotherms show no rapid initial uptake of adsorbent and occur when the forces of adsorption in the first monolayer are relatively small. These isotherms are rare. 


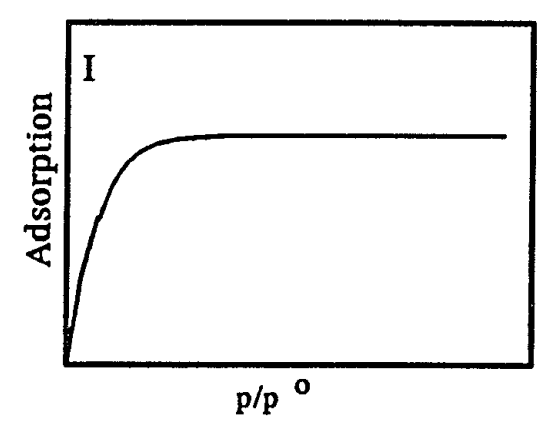

(A) Type I adsorption isotherm

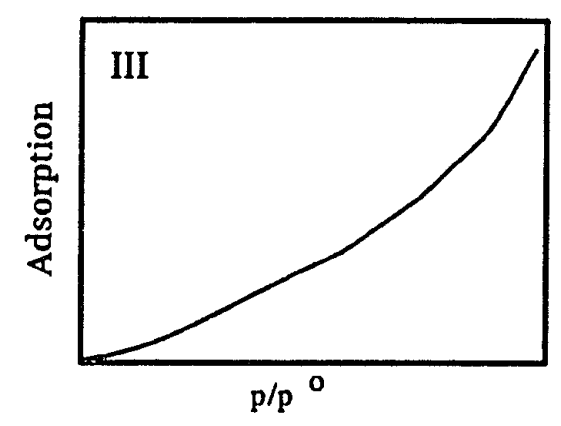

(C) Type III adsorption Isotherm

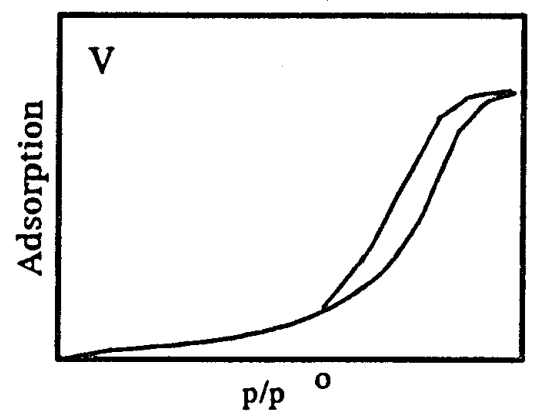

(E) Type V Adsorption Isotherm

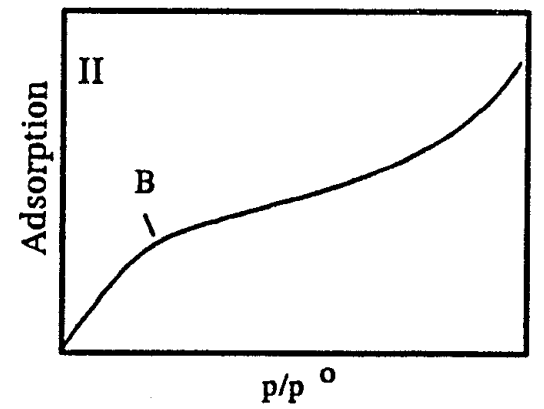

(B) Type II adsorption isotherm

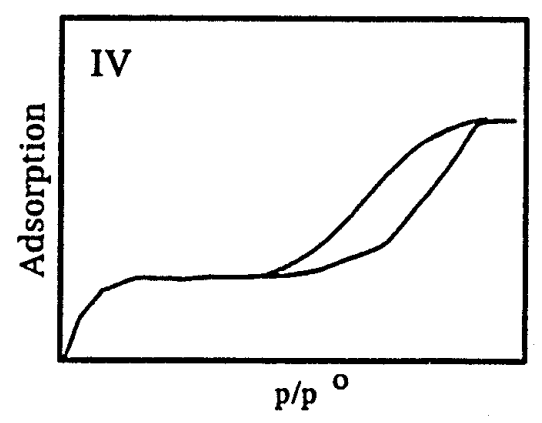

(D) Type IV adsorption Isotherm

Figure 6. Schematic diagram of BET adsorption isotherms 
Type IV isotherms level off near the saturation vapor pressure and are considered to reflect capillary condensation in porous solids. The upper limit of adsorption is mainly governed by the total pore volume. The isotherms of Type IV and $\mathrm{V}$ posses a hysteresis loop where the lower branch represents the addition of gas to the system and the upper branch withdrawal of gas from the system. Hysteresis effects can also appear in isotherms of Types I through III. It has been noted that there are a number of isotherms which can not be fit into any of the classifications.

The quantity (volume or mass) of gas taken up by a sample is proportional to the mass, $m$, of the sample. It also depends on temperature, $T$, the pressure, $p$, of the vapor and the nature of both the solid and the gas. If $n$ is the quantity of gas adsorbed expressed in moles/gram of solid, then

$$
n=f(p, T, \text { gas, solid })
$$

For a given gas adsorbed on a particular solid, maintained at a fixed temperature, Equation [3] reduces to

$$
n=f(p) T, \text { gas, solid }
$$

If the temperature is below the critical temperature of the gas an alternative expression for Equation [4] is

$$
n=f\left(p / p^{o}\right) T, \text { gas, solid }
$$

where

$$
p^{o}=\text { saturation vapor pressure of the adsorptive. }
$$


Equations [4] and [5] are simply expressions of the relationship between the amount of gas adsorbed, at constant temperature (adsorption isotherm), and the pressure or relative pressure of the adsorptive.

The physical adsorption of gases in the majority of cases gives rise to Type II isotherms. From the Type II isotherm, for any specific gas on a specific solid, it is possible to determine a value for the monolayer capacity of the solid which in turn can be used to calculate the specific surface of the solid. The monolayer capacity is defined as the amount of adsorbate which can be held in a completely filled single molecular layer on the surface of one gram of the solid.

The monolayer capacity, $n_{m}$ and specific surface area, $A$, are related by the equation

$$
A=n_{m} a_{m} L
$$

where

$$
a_{m}=\text { average area occupied by one molecule of }
$$

adsorbate, and

$$
L=\text { Avogadro's constant. }
$$

To obtain the monolayer capacity from the isotherms, it is necessary to interpret the Type II isotherm in quantitative terms. These treatments are well defined in the literature ${ }^{(35)}$ and are not repeated here. The best known treatment was by Brunauer, Emmett, and Teller (BET) which when applied has been successful in the evaluation of the specific surface area from a Type II isotherm. The BET treatment is based on a 
kinetic model of the adsorption process developed more than 60 years ago by Langmuir.(36) The BET equation is

$$
\frac{n}{n_{m}}=\frac{c\left(p / p^{o}\right)}{\left(1-p / p^{o}\right)\left(1+(c-1) p / p^{o}\right)}
$$

where

$$
\begin{array}{ll}
c & =e^{\left(q_{1}-q_{i}\right) / R T} \\
\left(q_{1}-q_{i}\right) & =\text { net heat of adsorption } \\
q_{1} & =\text { molar heat of condensation } \\
q_{i} & =\text { isosteric heat of adsorption }
\end{array}
$$

For convenience of plotting Equation [7] is often rewritten as

$$
\frac{p / p^{o}}{n\left(1-p / p^{o}\right)}=\frac{1}{n_{m} c}+\frac{c-1}{n_{m} c} \frac{p}{p^{o}}
$$

When $\frac{p / p^{o}}{n\left(1-p / p^{o}\right)}$ is plotted against $p / p^{o}$ the resulting straight line will have a slope of 


$$
\frac{c-1}{n_{m} c}
$$

with an intercept of

$$
\frac{1}{n_{m} c}
$$

From the slope and intercept the monolayer capacity, $n_{m}$, is given by

$$
n_{m}=1 /(\text { slope }+ \text { intercept })
$$

and the net heat of adsorption can be calculated from

$$
c=\text { slope } /(\text { intercept }+1)
$$

\subsection{Quartz crystal micro balance theory and operation}

The piezoelectric effect was discovered in 1880 by Jacques and Pierre Curie.(37) They found that a mechanical stress applied to the surfaces of some crystals produced a corresponding electrical potential across the crystal. The magnitude of this voltage was proportional to the applied stress. At the same time they observed that a voltage applied to certain crystals produced a strain in the crystal. This became known as the converse piezoelectric effect.

The definitions and application of piezoelectric materials involves the relationship between electric field, electric displacement, electric polarization, stress, and strain. The first three of these quantities are vectors while the last two are second rank tensors. A good introduction to the tensor formulation of physical properties can be found in Nye. ${ }^{(38)}$

The two reactions that occur in piezoelectrics can be written as 
where the field produced by stress is $\xi(\mathrm{V} / \mathrm{m}), \sigma$ is the applied stress $(\mathrm{Pa}), \varepsilon$ is the strain, and $g$ and $d$ are constants. The piezoelectric constant for some selected materials is shown in Table 2.

Table 2 - The Piezoelectric Constant of Various Materials

\begin{tabular}{lc}
\hline Material & $\begin{array}{c}\text { Piezoelectric Constant } \\
(\mathrm{m} / \mathrm{V})\end{array}$ \\
\hline Quartz & $2.3 \times 10^{-12}$ \\
$\mathrm{BaTiO}_{3}$ & $100 \times 10^{-12}$ \\
$\mathrm{PbZrTiO}_{6}$ & $250 \times 10^{-12}$ \\
$\mathrm{PbNb}_{2} \mathrm{O}_{6}$ & $80 \times 10^{-12}$ \\
\hline
\end{tabular}

The piezoelectric effect is very small. A field of $1000 \mathrm{~V} / \mathrm{cm}$ in quartz produces a strain of only about $10^{-7}$. This means a rod $1 \mathrm{~cm}$ long would change its length by 0.1 $\mathrm{nm}$ in the presence of a $1000 \mathrm{~V} / \mathrm{cm}$ field.

The converse piezoelectric effect can be used to construct a stable oscillator. If an alternating electric field is connected across a quartz crystal an alternating strain field will result. This causes a vibrational motion in the quartz crystal. Depending on various criteria the quartz oscillator will have a strong preference to vibrate at a characteristic resonant frequency.

As with all mechanical structures, a piezoelectric quartz crystal resonator can have many modes of resonance, or standing wave patterns at the resonant frequencies. As an example, a rectangular solid bar may exhibit three different types of vibrations: 
longitudinal, lateral (flexural and shear), and torsional. Coupling of modes and overtones can also produce other combinations. For controllable, repeatable performance the quartz crystal resonator should oscillate with only one principle mode. The selection of a particular mode and the suppression of all unwanted modes requires that the quartz crystal slab be cut at a specific crystallographic angle and have the proper shape.

Application of an alternating field across the thickness of an AT cut quartz crystal slab results in shear vibration in the $\mathrm{x}$-axis direction parallel to the electric field and propagation of a transverse shear wave through the crystal in the thickness direction. This is illustrated in Figure 7.

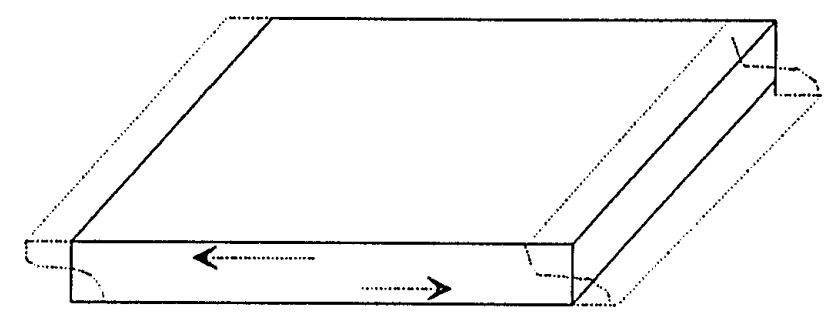

Figure 7. Thickness -shear mode of vibration

Due to its large use in electrical applications a Cartesian coordinate system was defined for quartz. The 1978 IEEE Standard on Piezoelectricity defines the Cartesian coordinates for $\alpha$ - quartz as follows:

(1) The $\mathrm{Z}$ axis is parallel to the $\mathrm{c}$ axis and the positive direction is arbitrary.

(2) The $X$ axis is chosen to lie along one of the three equivalent a axes. 
(3) The $Y$ axis is chosen to form a right- handed coordinate system for both the right and left handed quartz crystals.

Quartz crystal microbalances (QCM's) have been developed largely with a quartz crystal cut from the coordinate system defined above and shown in Figure 8 . This cut is known as the rotated y cut family and is specifically called an AT cut.

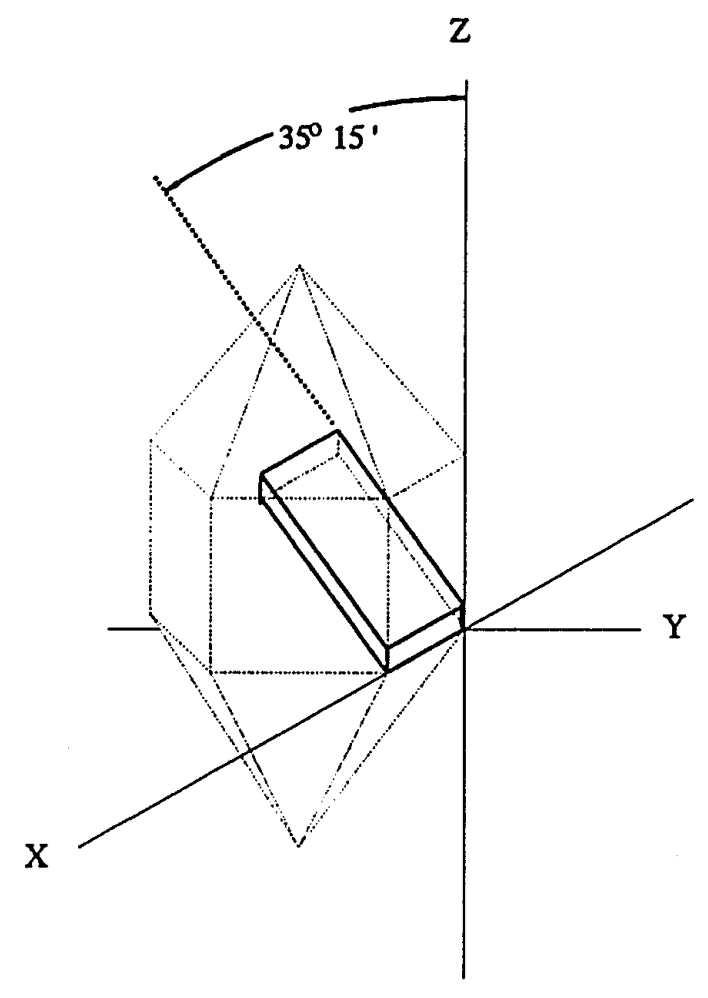

Figure 8. AT - cut quartz crystal

The role of the piezoelectric effect in mass detection is readily explained by the example of the thickness-shear mode exhibited by AT-cut quartz crystals. The shear 
motion that results from an alternating electric field applied at the electrodes is analogous to transverse waves traveling in a string of length $\mathrm{L}$. If we consider the string to be bound at both ends, standing waves of wavelengths which are integral divisors of 2L can result. From basic physics a resonant condition with standing waves is satisfied when the string is driven by a frequency matching the fundamental or one of the harmonic frequencies. The fundamental frequency $\left(f_{0}\right)$ of the string is $\left(S / m_{1}\right)^{1 / 2 / 2 L}$, where $S$ is the force (tension) on the string and $m_{l}$ is the mass per unit length

where

$$
\mathrm{f}_{\mathrm{O}}=\left(\mathrm{S} / \mathrm{m}_{\mathrm{l}}\right)^{1 / 2 / 2 \mathrm{~L}}
$$

An examination of Equation [13] shows that an increase in $\mathrm{m}_{\mathrm{l}}$ results in a decrease in the fundamental frequency of vibration.

In an AT-cut quartz crystal the fundamental shear mode can be shown as

$$
f_{o}=(u / \rho)^{1 / 2} / 2 t_{q}
$$

where $\mu$ is the shear modulus $\left(2.947 \times 10^{11}\right.$ dynes $\left./ \mathrm{cm}^{2}\right)$ and $\rho$ is the density $(2.648$ $\left.\mathrm{g} / \mathrm{cm}^{3}\right)$ of quartz. Increasing the quartz crystal thickness $\left(t_{\mathrm{q}}\right)$ results in an increase in wavelength $\left(\lambda=2 t_{q}\right)$ and a decrease in the fundamental frequency. If the mass can be increased without a change in the thickness of the crystal, the mass per unit length increases and the fundamental frequency decreases.

In 1957 Sauerbrey(39) provided a description and experimental proof of the mass-frequency relation for foreign layers deposited on thickness-shear mode (AT-cut quartz) crystals. This description was later modified by Miller and Bolef. ${ }^{(40)}$

Miller and Bolef treated the quartz film composite as a one dimensional compound acoustical resonator. Their results also indicated that the elastic properties 
of the deposited film should be related to the frequency shift. A thickness frequency equation was developed:

$$
T_{f}=\left(N_{q} d_{q} / I d_{f} f_{c} Z\right) \tan ^{-1}\left(Z \tan \left[\Pi\left(f_{q}-f_{c}\right) / f_{q}\right]\right)
$$

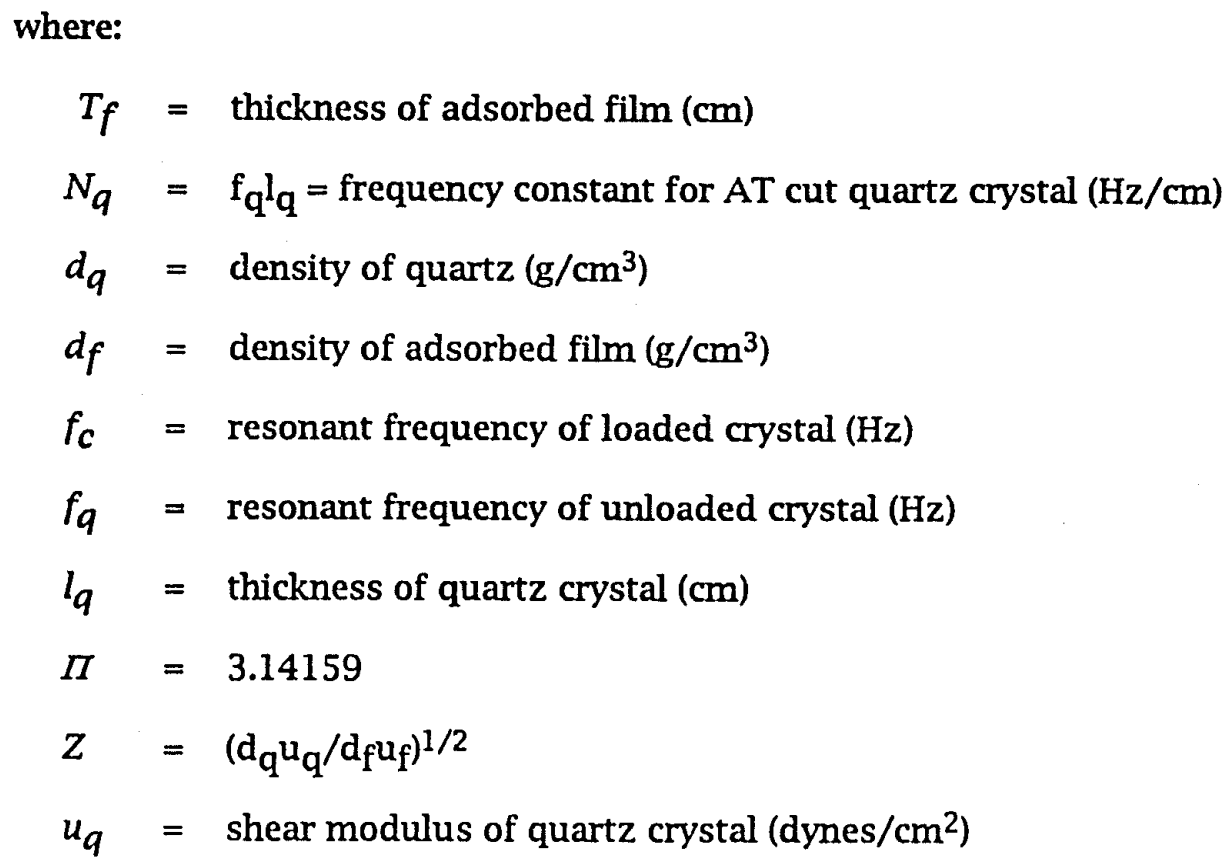

Equation [15] shows that the extent of frequency change is proportional to the mass of material adsorbed. The above mass frequency relationship has repeatedly been verified with controlled experiments. ${ }^{(41)}$ It is therefore not necessary to calibrate quartz microbalance wafers empirically.

Thermal expansion is a property that directly affects crystal resonator performance. The frequency versus temperature characteristics of a resonator are determined almost entirely by the temperature dependence of the elastic constants and the thermal expansion of the crystal. 
Figure 9 shows that the frequency change of an oscillating AT-cut quartz crystal depends not only on the deposited mass but also on the temperature. As can be seen, very small temperature coefficients can be obtained near room temperature for a cutting angle of $35^{\circ} 10^{\prime}$. Since frequency changes caused by temperature changes simulate mass loading and hence could lead to errors in mass thickness determinations, heating of the quartz crystal has to be prevented as much as possible. Temperature changes are minimized by water jackets.

In the deposition of highly stressing films such as chromium, silicon, titanium, and silica, the mechanical stress itself can be transmitted through the exciting electrode of the crystal and can bend the crystal.(42) Bending of the crystal, which can also occur by improper mounting in the holder, tends to decrease the crystal activity and promote spurious secondary modes of oscillation.

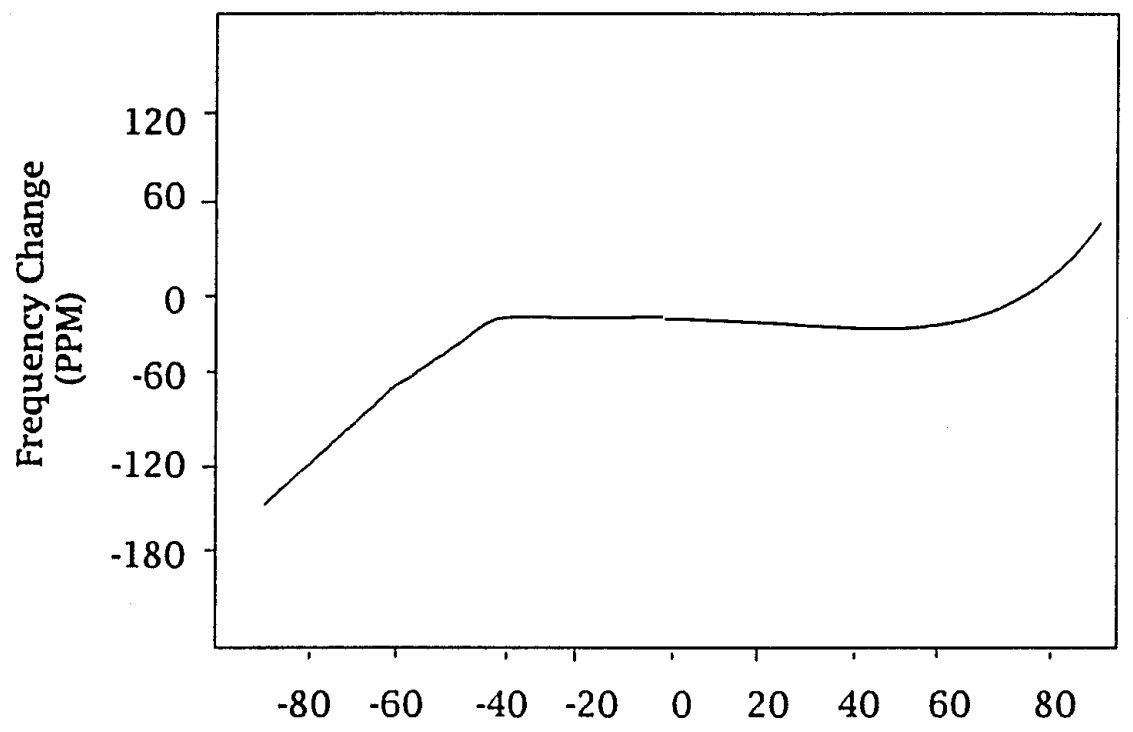

Temperature $\left({ }^{0} \mathrm{C}\right)$

Figure 9. Frequency change vs. temperature for an AT crystal cut at $35^{\circ} 15^{\prime}$ 
In practice, the accuracy of quartz crystal monitors is determined by the stability of the oscillator circuit. If a frequency change of $1 \mathrm{~Hz}$ in a $5 \mathrm{Mhz}$ oscillator can be detected, the corresponding sensitivity of the micro balance is about $2 \times 10^{-8}$ $\mathrm{g} / \mathrm{cm}^{2}$. With proper temperature control ${ }^{(43)}$ the sensitivity can be improved to $10^{-10}$ to $10^{-11}$ grams.

Among the first applications of the quartz crystal microbalance (QCM) was the study of the physical adsorption of gases. Slutsky and Wade ${ }^{(44)}$ used a crystal mounting that allowed gas to adsorb directly onto crystalline quartz. A similar system was used by $\mathrm{Kahn}^{(45)}$ to investigate the isotherms for argon, hexane, and water vapor on the bare surface of a quartz crystal. No claim to clean quartz surfaces could be made by these authors since high vacuum conditions were not met and the surfaces of the quartz crystals were not cleaned in situ. These and similar experiments demonstrated that the QCM could be of value in studies of physical adsorption.

The adsorption of water on evaporated gold surfaces on a QCM was investigated by Thomas and Sharma.(46,47) The gold surfaces were not deposited under UHV conditions. The films were also exposed to water vapor before adsorption and desorption isotherms were developed. The BET plots of the adsorption isotherms indicated that the net heat of adsorption of water on water-covered gold is about 4.2 $\mathrm{kJ} / \mathrm{mole}$.

In a study of the atmospheric corrosion of cobalt, Rice and co-workers ${ }^{(48)}$ studied the adsorption of moisture on cobalt surfaces as a function of relative humidity. By using the QCM they were able to establish relationships which served as the cornerstone for the development of cobalt based alloys used in magnetic recording.

The above review of the theory of QCM's shows a large amount of research has been put into their understanding and development. With the proper control of 
variables a micro weighing system can be developed which will accurately measure $1 \mathrm{x}$ $10^{-11}$ grams. 


\section{CHAPTER 3}

\section{Research Objectives and Hypothesis}

Any solid is capable of adsorbing a certain amount of gas or vapor. The extent of this adsorption at equilibrium will depend on temperature, the pressure of the gas, the effective surface area of the solid, and the reactivity of the surface.

The surface reactivity of carbon films, to a large extent, is determined by the bonding characteristics. The increased $s p^{2}$ characteristics of the sputtered graphitic films as opposed to the increased $s p^{3}$ characteristics of the PECVD films, will lead to a higher imbalance of attractive forces at the surface. This type of surface can be expected to adsorb more of a polar molecule such as $\mathrm{H}_{2} \mathrm{O}$. Adsorption reduces the imbalance of bonding forces which exist at the surface and therefore the surface free energy of the system. The PECVD films with a higher $s p^{3}$ bond content are therefore expected to adsorb less moisture.

The main objective of this study was to investigate the characteristics of moisture adsorption on carbon thin films used as magnetic disk overcoats. This required the development of a quartz crystal microbalance technique and control of the experimental variables to obtain a mass sensitivity of one nanogram. 


\section{CHAPTER 4}

\section{Experimental Methodology}

\subsection{Experimental overview}

The experimental work undertaken can be divided into six major categories, namely:

1) Construction of an environmental chamber containing temperature and humidity controls and a quartz crystal microbalance.

2) Determination of moisture adsorption on the gold electrodes of quartz crystals to evaluate chamber stability and develop experimental techniques.

3) DC magnetron sputtering of carbon on to the gold electrodes of a quartz crystal. Sputtering was done on at least two crystals at 25, 50 and $100 \mathrm{~nm}$ carbon thickness to allow experimental error determinations. At the same time carbon was sputtered on a silicon wafer for hardness and resistivity measurements.

4) Plasma enhanced chemical vapor deposition of carbon on the gold electrodes of a quartz crystal. Duplicate crystals at 50 and $100 \mathrm{~nm}$ carbon thickness were prepared to provide multiple samples for the determination of experimental error. Under identical conditions PECVD carbon was deposited onto a silicon wafer for evaluation of hardness and resistance.

5) Determination of the hardness of the carbon deposited on the silicon wafers by DC magnetron sputtering and by PECVD using a NANOII ${ }^{\circledR}$ hardness tester. The conductivity of each type of carbon was evaluated using a standard 4 point probe technique. 
6) Determination of adsorption isotherms by placing the carbon coated quartz crystals in the environmental chamber and measuring the amount of adsorbed moisture as a function of the relative humidity of the chamber at constant temperature.

The details for each of the above categories are contained in the sections that follow.

\subsection{Adsorption apparatus}

A schematic diagram of the apparatus used for the adsorption studies is shown in Figure 10. A clean-dry-air (CDA) source was used to supply air to a small aluminum environmental chamber. The chamber measurements were 24 inches wide by 10 inches in depth and six inches high with a hinged Plexiglas cover. The CDA source was split at a tee to provide two lines leading into the chamber. One line was routed through a one liter flask of DI water. The air volume in both lines was controlled by two King Instruments air flow controllers. The relative humidity was set by varying the volume of CDA going through the bubbler versus the volume of CDA going directly into the chamber. A front view photograph of the system is shown in Figure 11 and a rear view photograph displaying the DI water flask is shown in Figure 12.

The Inficon XTC/2 quartz crystal microbalance was connected through a sealed inlet in the upper one inch of the chamber. The quartz crystal was mounted in a Leybold-Inficon standard crystal holder, part number 007-083. An exploded schematic of the crystal and holder is shown in Figure 13. The crystal was mounted with the primary exposed surface facing down. The quartz crystal temperature was controlled through a Neslab RTE 9 refrigerated bath circulator with a Neslab DCR 1 digital controller. The resolution of the controller bath combination was $0.1^{\circ} \mathrm{C}$ and it was monitored to $\pm 0.1^{\circ} \mathrm{C}$ on the chart recorder. 


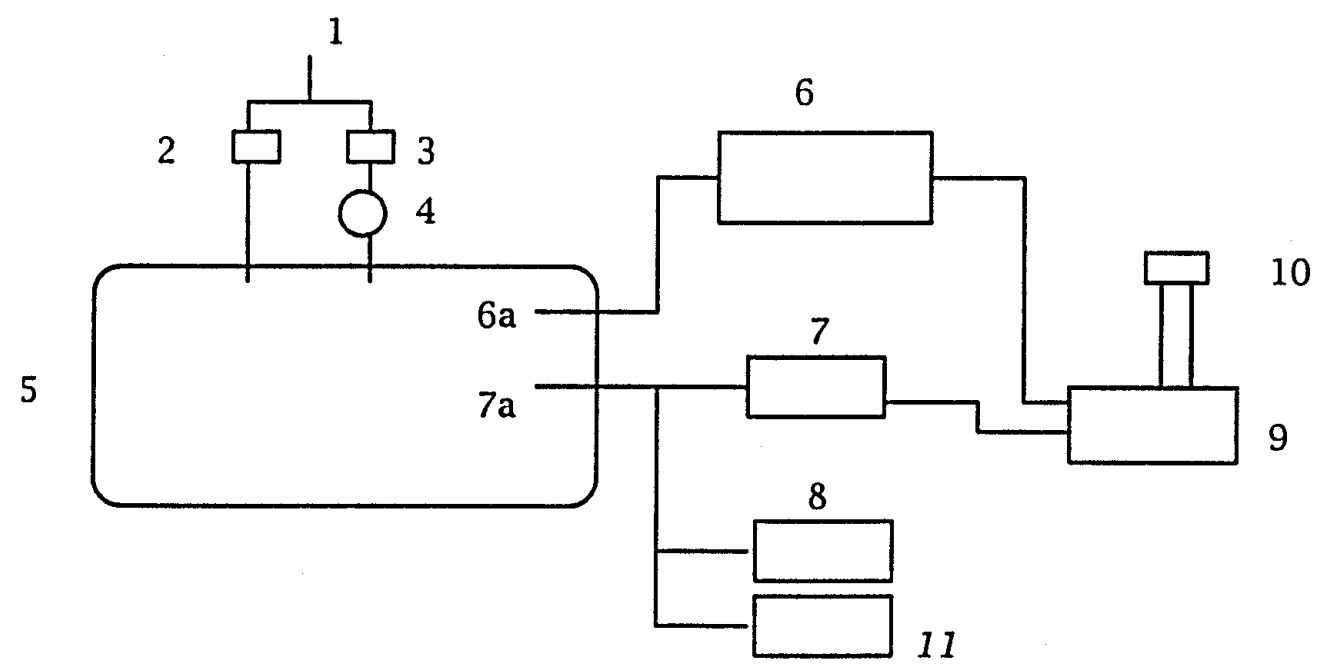

1. Clean dry air inlet

2. King Instruments air flow controller

3. King Instruments air flow controller

4. One liter DI water bubbler

5. Aluminum environmental chamber

6. Vaisal HMP131Y relative humidity and temperature transmitter

6a. Vaisal HMP131Y relative humidity and temperature probe

7. Inficon $X T C / 2$ quartz crystal rate monitor

7a. Inficon $6 \mathrm{Mhz}$ Au-quartz crystal (Part number 008-010-G10)

8. Neslab RTE 9 refrigerated bath circulator

9. Astro Med Dash IV multi channel chart recorder

10. Fluke 73 multimeter

11. Neslab DCR - 1 digital controller/readout

Figure 10. Schematic diagram of adsorption apparatus 


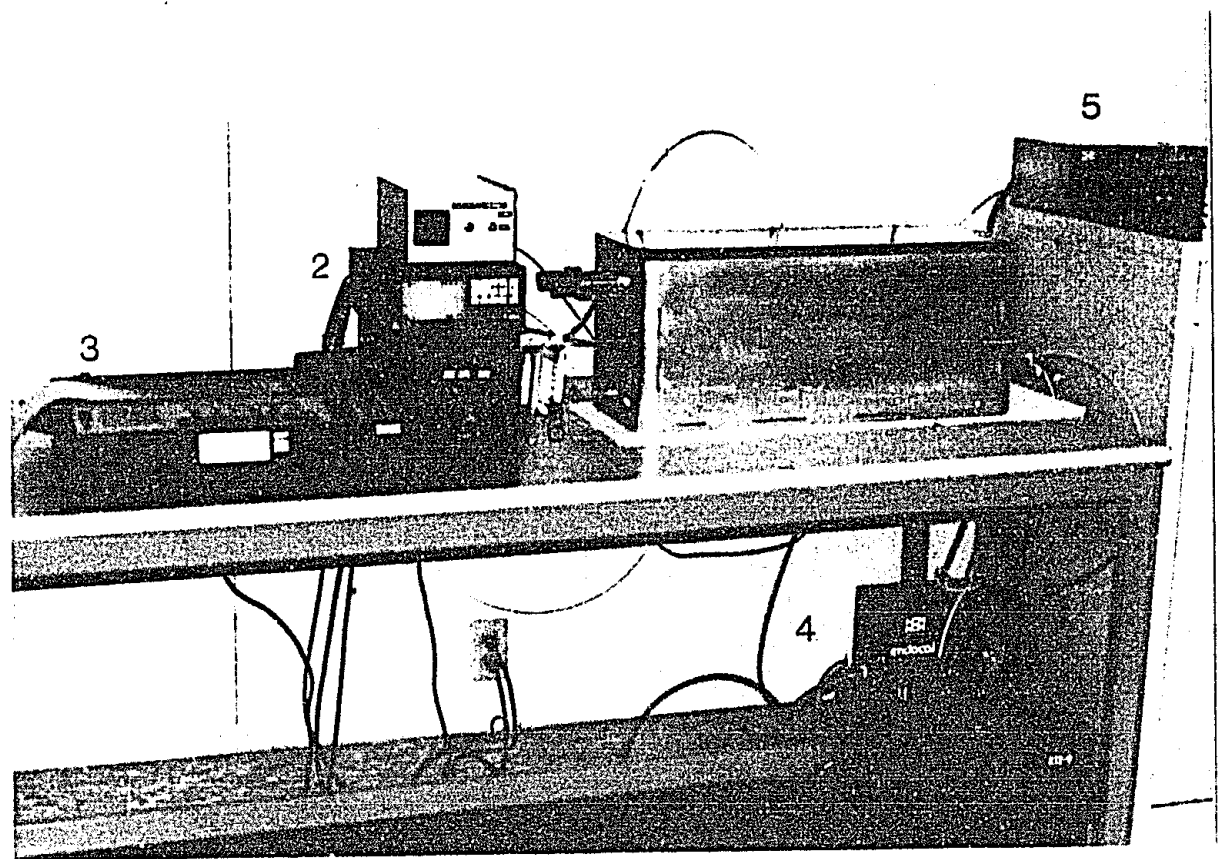

1. Aluminum environmental chamber

2. Inficon $X T C / 2$ quartz crystal rate monitor

3. Astro Med Dash IV multi channel chart recorder

4. Neslab RTE 9 refrigerated bath controller

5. Neslab DCR-1 digital controller and readout

6. King instruments air flow controller

Figure 11. Front view photograph of the adsorption apparatus 


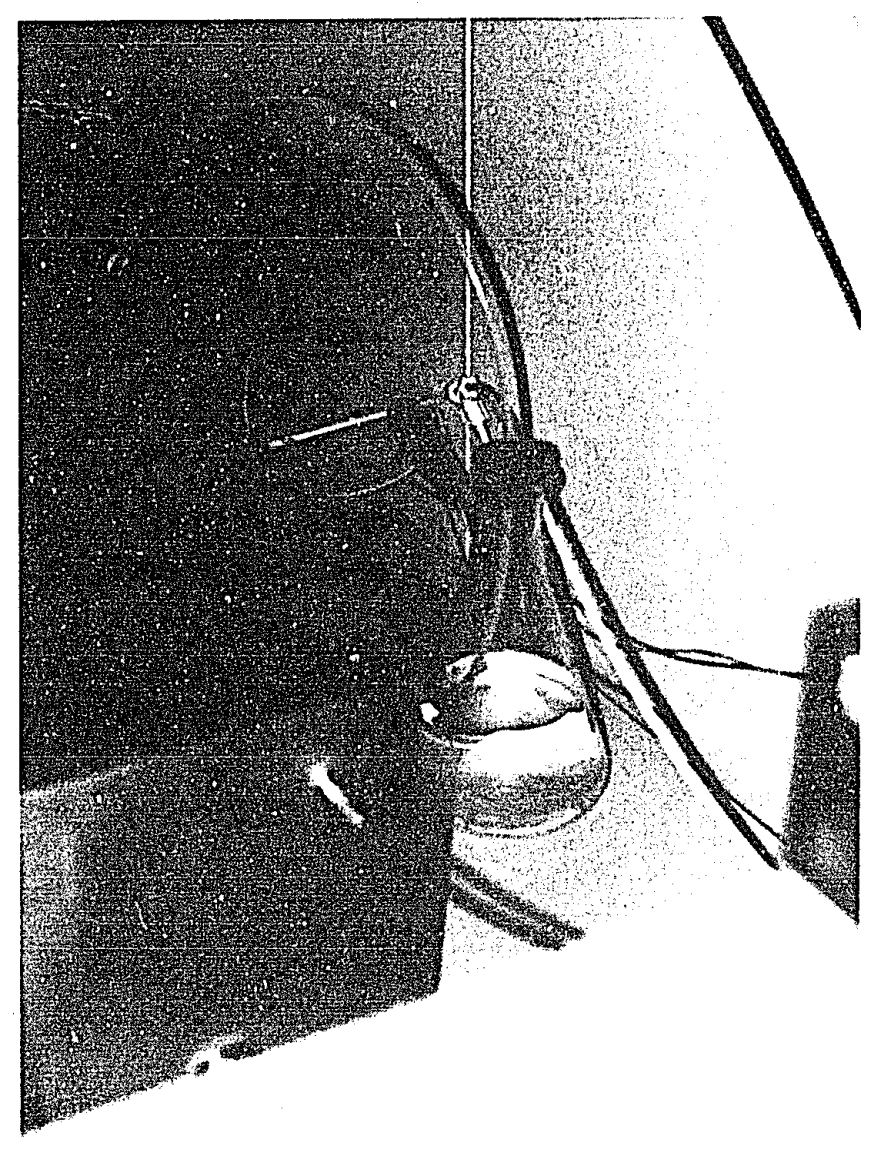

Figure 12. Rear view photograph of the adsorption apparatus 


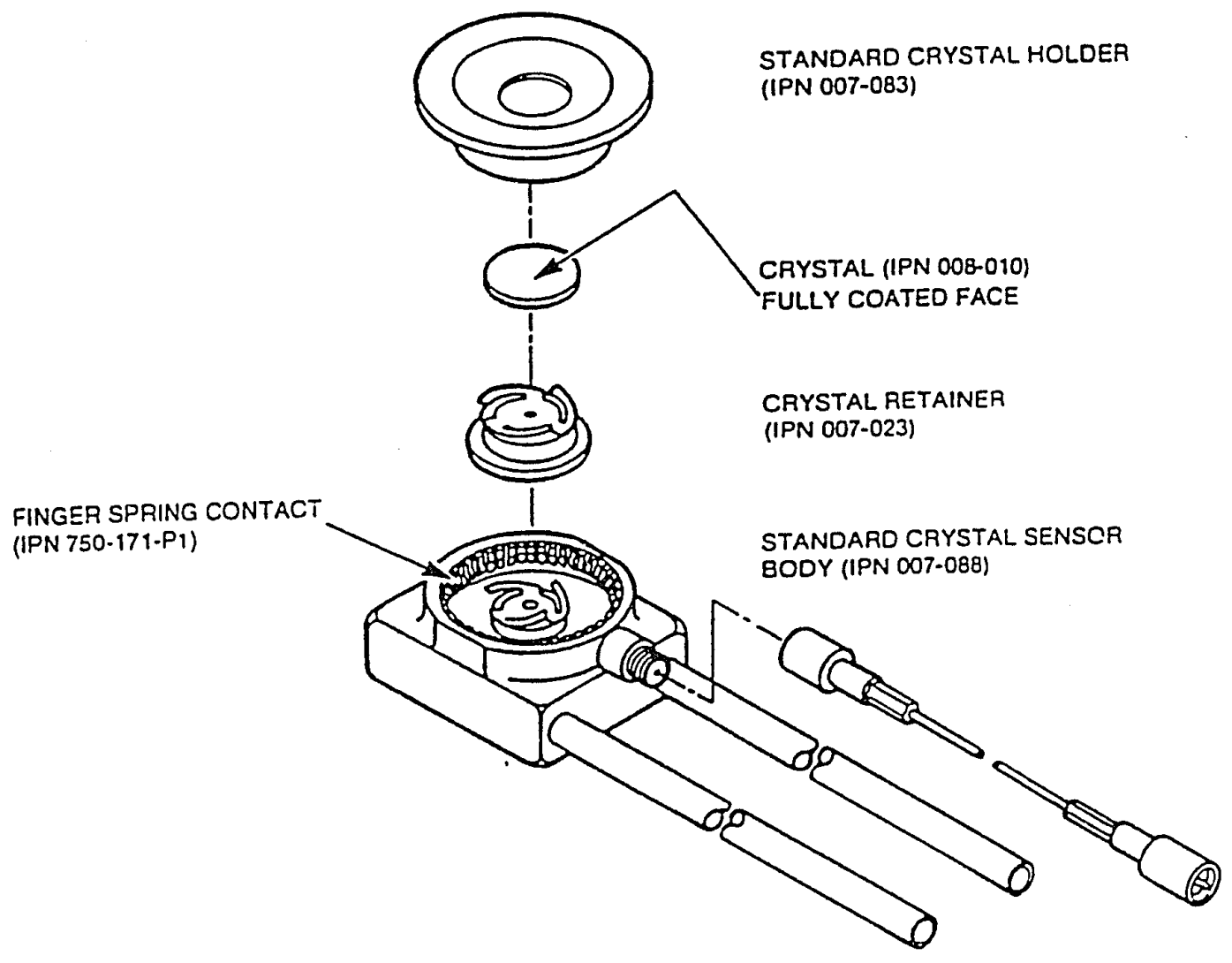

Figure 13. Leybold-Inficon standard crystal sensor 
The relative humidity was monitored with a Vaisal HMP $135 \mathrm{Y}$ relative humidity and temperature transmitter. The HUMICAP $\mathrm{H}$ sensor works by monitoring the change in capacitance of a thin polymer film as it adsorbs water vapor. The accuracy including non-repeatability, hysteris and calibration uncertainty was 1\% RH between zero and $90 \% \mathrm{RH}$. The response time was 15 seconds per reading. The stainless steel sensor body with a sintered bronze filter was sealed in the chamber and connected to the transmitter through a cable. The temperature accuracy of the Vaisal HMP 135Y relative humidity and temperature sensor was $\pm 0.1^{\circ} \mathrm{C}$ over the temperature range of 0 to $160^{\circ} \mathrm{C}$.

The Inficon $X T C / 2$ is a quartz crystal transducer deposition process controller. It has a $6 \mathrm{Mhz}, \pm 0.05 \mathrm{~Hz}$ per $250 \mathrm{msec}$ sample, to $5.0 \mathrm{Mhz}, \pm 0.05 \mathrm{~Hz}$ per $250 \mathrm{msec}$ sample, crystal range and precision. This translates to a sample thickness resolution of $0.0617 \AA$ per $250 \mathrm{msec}$ sample. With a crystal holder opening of approximately 0.527 $\mathrm{cm}^{2}$, a material with a density of one will be detectable to an accuracy of \pm 0.62 nanograms $/ \mathrm{cm}^{2}$. The $\mathrm{XTC} / 2$ was factory programmed to read in angstroms.

The XTC/2 was connected to an Astro Med Dash IV four channel chart recorder. The chart was calibrated to one angstrom moisture adsorption with a full scale reading of $100 \mathrm{~A}$. The other three channels were used to monitor percent relative humidity, chamber temperature and crystal temperature.

The chamber was heated with 3 Watlow resistance cartridge heaters (part number G4A40) measuring $1 / 4$ inch in diameter and 4 inches in length. The heaters were sealed in the base plate of the chamber and connected to an Omega CN9000A controller with a $0.1^{\circ} \mathrm{C}$ temperature resolution. 


\subsection{Evaluation of moisture adsorption on gold electrodes of quartz crystals}

Quartz piezoelectric crystals with gold electrodes were obtained from LeyboldInficon (Part number 008-010-G10). Prior to carbon deposition each quartz crystal was immersed in Freon TF vapor for three minutes then slowly withdrawn from the vapor over a one minute period. The crystals were immediately placed in glass petri dishes, sealed with a glass cover, and held for adsorption studies.

A quartz crystal was inserted in the Inficon crystal holder and placed in the environmental chamber for moisture adsorption evaluations on the factory deposited gold electrodes. The CDA was opened to a flow rate of 9 standard cubic feet per hour $(\mathrm{SCFH})$ and the $\mathrm{RH}$ was reduced to $0 \%$. In approximately one hour the adsorbed thickness of moisture on the crystal was stable to $\pm 1 \mathrm{~A}$ After a stable thickness was obtained, the crystals were held an additional 15 minutes at $\mathrm{O} \% \mathrm{RH}$ before the adsorption isotherms were determined. Two adsorption isotherms were run on each crystal by varying the $\mathrm{RH}$ from 0 to $70 \%$ and then back down to $0 \%$. After one hour of stable weight, the air flow controller valve controlling flow through the DI water bubbler was opened to $6 \mathrm{SCFH}$ and the air flow controller admitting CDA to the chamber was closed. The relative humidity of the chamber was brought to $70 \%$ within $9-10$ minutes and held at $70 \% \mathrm{RH}$ for 3-10 minutes. The CDA through the DI water was stopped and the CDA to the chamber was opened to 6 SCFH. The RH was reduced to $10 \%$ within 15 minutes and to $0 \%$ within one hour. An identical procedure was used for each crystal. Twelve crystals were evaluated, with eleven having very similar adsorption isotherms. One crystal showed $80 \%$ less moisture adsorption despite a second degreasing in Freon TF. No further evaluations were performed with the low adsorption crystal.

A series of experiments were performed on the gold electrode quartz crystals to determine if isothermal equilibrium adsorption thickness was affected by the rate at 
which the relative humidity was either increased or decreased. This was accomplished by varying the time to increase relative humidity from 5 minutes to 1 hour for a change from 0 to $70 \% \mathrm{RH}$. A 70\% $\mathrm{RH}$ was also maintained for 72 hours with the adsorbed thickness remaining within $\pm 1 \AA$.

After the adsorption evaluations, the eleven selected crystals were vapor degreased, as described above, placed in glass petri dishes, and held for carbon deposition.

\subsection{Plasma enhanced chemical vapor deposition of carbon on gold electrodes of quartz crystals}

One quartz crystal was placed on the cathode plate of a Samco BP-1 Plasma Deposition System. A typical plasma deposition system is schematically illustrated in Figure 14. One half of the crystal was covered with a glass cover slide. After the sample chamber pressure was reduced to approximately 5 mtorr a nitrogen purge was established at 120 mtorr. After 10 additional minutes the nitrogen purge was stopped and the system evacuated to one mtorr. Argon was admitted into the chamber at a pressure of 120 mtorr and a $13.56 \mathrm{Mhz}$ plasma established so that a $600-800 \mathrm{~V}$ bias was measured on the cathode. A carbon film was deposited on the quartz crystal by immediately admitting vapor from a degassed liquid hydrocarbon into the evacuated reactor while shutting down the argon. Deposition was carried out at $800 \mathrm{~V}$ bias on the cathode and a gas pressure of 30 mtorr. The deposition was maintained for 30 seconds, at which time the crystal was removed and the thickness measured on a Dektak 3030 profilometer by comparing the deposited area height to the non-deposited area shielded by the glass slide. The deposition rate was calculated by dividing the film thickness by the deposition time. 


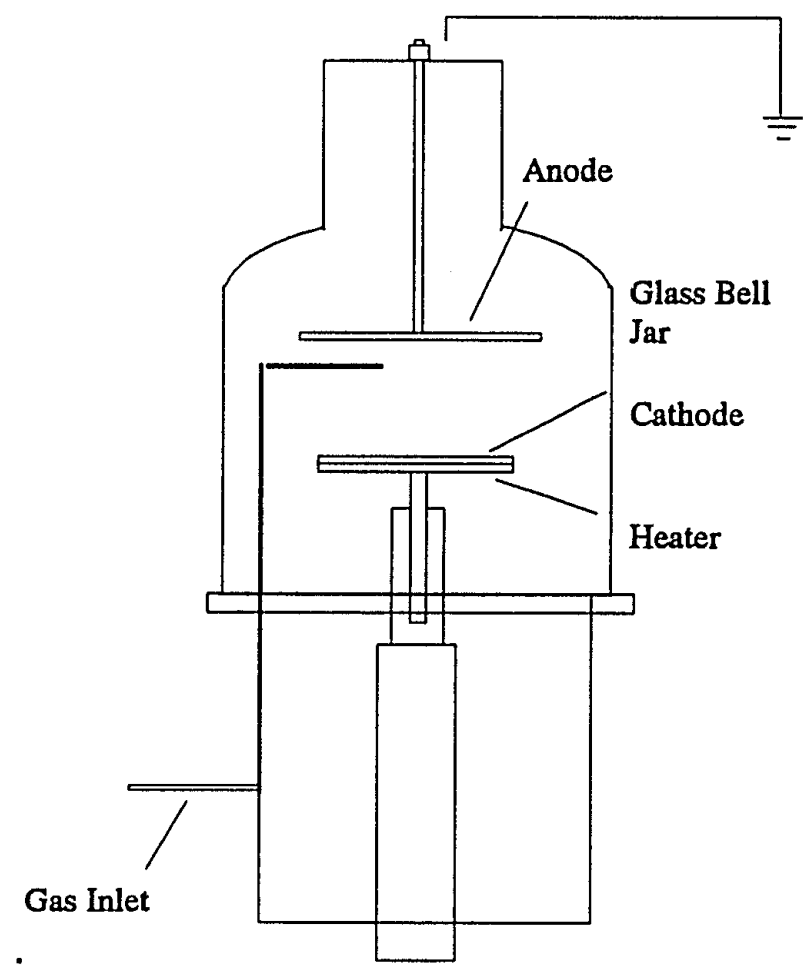

Figure 14. PECVD system

A quartz crystal which was to be used for the evaluation of moisture adsorption was placed in the Leybold-Inficon crystal holder shown in Figure 12. The crystal holder limited the carbon deposition to the $0.527 \mathrm{~cm}^{2}$ circular opening in the cover plate of the crystal. By this technique the carbon could be limited to only the active area of the crystal. One crystal was placed on the cathode of the Samco BP-1 deposition system and carbon was deposited through the circular opening for 15 seconds using the procedure described above. After venting the vacuum to atmospheric pressure the sample was removed from the crystal holder and replaced with the opposite side exposed. The crystal was put in the PECVD system and carbon was deposited on the opposite surface for 15 seconds. This procedure was repeated for another crystal. A 
third and fourth crystal had carbon deposited on both surfaces for 30 seconds per side thus giving a total of $100 \mathrm{~nm}$ of carbon on each surface of each crystal.

\subsection{Deposition of DC magnetron sputtered carbon on gold electrodes of quartz crystals}

Sputtered carbon films were prepared using a DC magnetron sputtering system which is schematically shown in Figure 15. A two inch diameter graphite target (Tosoh, 99.9\% C) was attached to the cathode of the sputtering system and used as the carbon source. The distance between the cathode and the quartz crystal surface was approximately four inches. A quartz crystal with one half of a side covered by a glass slide was placed on the anode of the system. The chamber pressure was reduced to $1 \mathrm{x}$ $10^{-6}$ torr for 15 minutes. Argon was admitted to the discharge chamber and a $500 \mathrm{~V}$ DC voltage was used to ionize the gas at a total pressure of 20 mtorr. The deposition rate was determined to be $10 \mathrm{~nm}$ per minute by the step profile technique used for the PECVD carbon.

The crystals which were to be used for evaluation of moisture adsorption were placed in the Leybold-Inficon crystal holder to limit the deposition to the previously measured $\left(0.527 \mathrm{~cm}^{2}\right)$ open circular area of the crystal holder. Two crystals had carbon deposited for 150 seconds each for a thickness of $25 \mathrm{~nm}$. Three crystals had carbon deposited at separate times for 300 seconds each for a total thickness of $50 \mathrm{~nm}$. Two more crystals had carbon deposited on them for 600 seconds each for a total carbon thickness of $100 \mathrm{~nm}$. The crystals were carefully removed from the crystal holder after each deposition and replaced in the holder with the opposite surface showing.

The carbon target was removed from the DC magnetron sputtering system and a $\mathrm{Co}_{76} \mathrm{Cr}_{10} \mathrm{Ta}_{4}$ (Cerac, 99.9\%) alloy target was installed in the cathode position. A small 


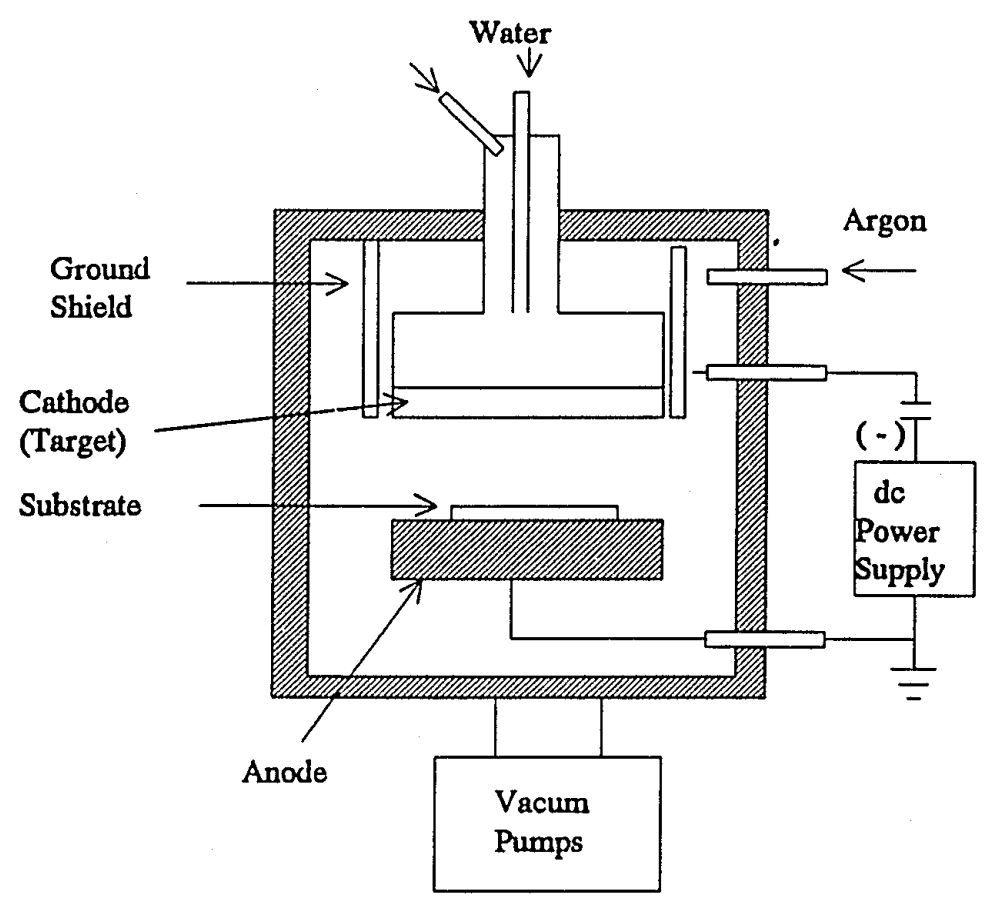

Figure 15. Schematic drawing of a DC magnetron sputtering system

glass slide was placed in the anode position with one half of the glass slide covered by another slide. The chamber pressure was reduced to $1 \times 10^{-7}$ torr for 15 minutes. Argon was admitted to the discharge chamber and a $600 \mathrm{~V} \mathrm{DC}$ voltage was used to ionize the gas at a total pressure of 20 mtorr. The magnetic alloy was sputtered for 4 minutes at which time the voltage was reduced to 0 and the system vented to atmospheric pressure. The thickness of the magnetic alloy deposited on the glass slide was again measured on a Dektak 3030 profilometer. The deposition rate was determined as $24.2 \mathrm{~nm}$ per minute.

A quartz crystal was installed in the Leybold-Inficon crystal holder and placed on the anode of the sputtering system. Fifty $\mathrm{nm}$ of cobalt alloy was deposited on the exposed surface of the crystal using the above conditions by sputtering for 2 minutes and 4 seconds. The crystal was reversed in the sample holder and the opposite surface 
sputtered in the same manner. After deposition with the magnetic alloy the cobalt alloy target was replaced with the previously used graphitic carbon target. Fifty nm of carbon was deposited on both faces of the crystal using the previously defined carbon sputtering conditions.

\subsection{Adsorption of moisture on carbon}

Prior to moisture adsorption studies each carbon coated quartz crystal was immersed in Freon TF vapor for three minutes than slowly withdrawn from the vapor over a one minute period. The crystals were immediately placed in glass petri dishes, sealed with a glass cover, and held.

A quartz crystal with $50 \mathrm{~nm}$ of DC magnetron carbon on each surface was inserted in the Inficon crystal holder and placed in the environmental chamber for moisture adsorption evaluations. The CDA was opened to a flow rate of 9 standard cubic feet per hour (SCFH) and the $\mathrm{RH}$ was reduced to 0\%. In approximately one hour the adsorbed thickness of moisture on the crystal was stable to \pm 1 A. After a stable thickness was obtained the crystals were held an additional 15 minutes at O\% RH before the adsorption isotherms were measured. Two adsorption isotherms were run on each crystal by varying the RH from 0 to $70 \%$ and then back down to $0 \%$. After one hour of stable weight, the air flow controller valve controlling flow through the DI water bubbler was opened to $6 \mathrm{SCFH}$ and the air flow controller admitting CDA to the chamber was closed. The relative humidity of the chamber was brought to $70 \%$ within $9-10$ minutes and held there for 3-10 minutes. The CDA through the DI water was stopped and the CDA to the chamber was opened to $6 \mathrm{SCFH}$. The RH was reduced to $10 \%$ within 15 minutes and to $0 \%$ within one hour. An identical procedure was used for each crystal. 
A series of experiments were performed on the carbon coated quartz crystals to determine if the adsorption thickness was affected by the amount of time for either the adsorption or desorption process. This procedure was identical to the procedure performed previously on the gold electrodes.

\subsection{Film characterization}

At the time of the depositions described earlier identical films of $100 \mathrm{~nm}$ thickness were deposited on three inch silicon wafers. In the case of DC magnetron sputtering carbon was deposited on the silicon wafer in the same deposition as the piezoelectric crystal. In the case of the PECVD carbon was deposited with the same conditions but separately.

The electrical resistance, $R_{S}$, of the film was measured using a four point probe method.(49) In this method a current $I$ is fed in through any two adjacent probes and the voltage $V$ generated across the other two is measured. The sheet resistance is then computed from

$$
\mathrm{R} s=\frac{V}{I} \frac{2 \Pi}{\ln 2}=9.06 \frac{V}{I}
$$

The silicon wafers with the PECVD and DC magnetron deposited carbon were analyzed for hydrogen content by hydrogen forward scattering (HFS) spectrometry at Charles Evans, Redwood City, CA. HFS employs the same experimental components as those used in conventional Rutherford backscattering spectrometry. The primary differences are the grazing incidence arrangement of the He beam, sample and detector and the addition of an attenuator foil in front of the detector.

The hardness of the films was determined with a Nanoindenter II (Nano Instruments). In this measurement, the capacitance displacement gauge detects the 
plastic depth with an accuracy of $0.2-0.3 \mathrm{~nm}$. The force resolution of the system is about $0.5 \mu \mathrm{N}$. The following experimental conditions were used: indentation rate $=10$ $\mathrm{nm} / \mathrm{s}$, holding time $=5 \mathrm{~s}$, penetration depth $=50 \mathrm{~nm}$ and temperature $=25^{\circ} \mathrm{C}$. From load displacement curves, the contact deformation depth, $h_{p}$, and the elastic recovery depth, $h_{c}$, can be measured. The hardness, $H$, is defined as the average pressure under the indenter, namely:

$$
H=P / A
$$

where $P$ is the contact load. $A$, the contact area, is calculated from the known geometry of the indenter and $h_{p}$, according to the following equation:

$$
A=24.56 h_{p}^{2}+225.94 h_{p}^{3 / 2}+519.61 h_{p}
$$




\section{CHAPTER 5}

\section{Results and Discussion}

\subsection{Carbon Analysis}

In Chapter 1 it was shown that thin carbon films deposited by both PECVD and DC magnetron sputtering can have widely varying properties. In some cases, as was shown in Table 2, these properties can overlap. To characterize the carbons used in this study, samples were deposited on silicon wafers by both DC magnetron sputtering and PECVD during the depositions on the quartz crystals. These samples were measured for hardness, hydrogen content, and resistivity and the results are shown in Table 3 . As expected from previously cited literature sources, the carbon deposited by PECVD exhibited higher hardness, higher resistivity, and higher hydrogen content than the DC magnetron sputtered carbon.

Table 3 - Carbon analysis: PECVD versus DC magnetron sputtered

\begin{tabular}{lccc}
\hline Deposition Process & $\begin{array}{c}\text { Hardness } \\
\text { (Gigapascals) }\end{array}$ & $\begin{array}{c}\text { Hydrogen } \\
\text { (Atomic \%) }\end{array}$ & $\begin{array}{c}\text { Resistivity } \\
(\Omega-\mathrm{cm})\end{array}$ \\
\hline PECVD & 22 & 27 & $3 \times 10^{11}$ \\
DC Magnetron & 14 & 4 & $7 \times 10^{4}$ \\
\hline
\end{tabular}

Previous authors ${ }^{(50)}$ have shown, through electron energy loss spectra (EELS) and Raman spectroscopy, that PECVD carbon films have a higher concentration of $s p^{3}$ bonding than DC magnetron sputtered carbon films. These studies, summarized in Table 4, had a hydrogen concentration, hardness, and resistivity, for both the PECVD and DC magnetron deposited carbon, similar to the samples produced in this investigation. 
Table 4 - Carbon properties: PECVD versus DC magnetron sputtered

\begin{tabular}{lccc}
\hline Deposition Process & $\begin{array}{c}\text { Hardness } \\
\text { (Gigapascals) }\end{array}$ & $\begin{array}{c}\text { Hydrogen } \\
(\%)\end{array}$ & $\begin{array}{c}\text { Resistivity } \\
(\Omega-\mathrm{cm})\end{array}$ \\
\hline PECVD & 33 & 18 & $>10^{6}$ \\
DC magnetron & 14 & 9 & $1.3 \times 10^{3}$ \\
\hline
\end{tabular}

The experimental evaluations of hardness, hydrogen content, and resistivity firmly established that the carbon films produced for this study were significantly different in structure from each other. A comparison with published studies of carbon verified that the PECVD carbon had significantly more $s p^{3}$ bond character than the DC magnetron sputtered carbon.

\subsection{Moisture adsorption on gold electrodes of quartz crystals}

The results of moisture adsorption on the gold electrodes of the piezoelectric crystals are shown in Figure 16. The adsorption isotherm shows Type III characteristics. The major distinguishing feature is a slight convexity towards the relative humidity axis commencing at the origin.

Type III isotherms are characteristic of weak gas-solid interactions.(51) The weakness of the adsorbent-adsorbate forces will cause the uptake at low relative pressures to be small, but once a molecule has become adsorbed, the adsorbateadsorbate forces will promote the adsorption of further molecules so that the isotherms will become convex to the pressure axis. Previous studies ${ }^{(52)}$ have shown that water can produce Type III isotherms because its London dispersion interaction energy is unusually small compared with its polar energy contribution. Water has provided many examples of Type III isotherms.

While Figure 16 does exhibit Type III adsorption isotherm characteristics this distinction is not perfectly clear. It is possible that some Type II characteristics also 

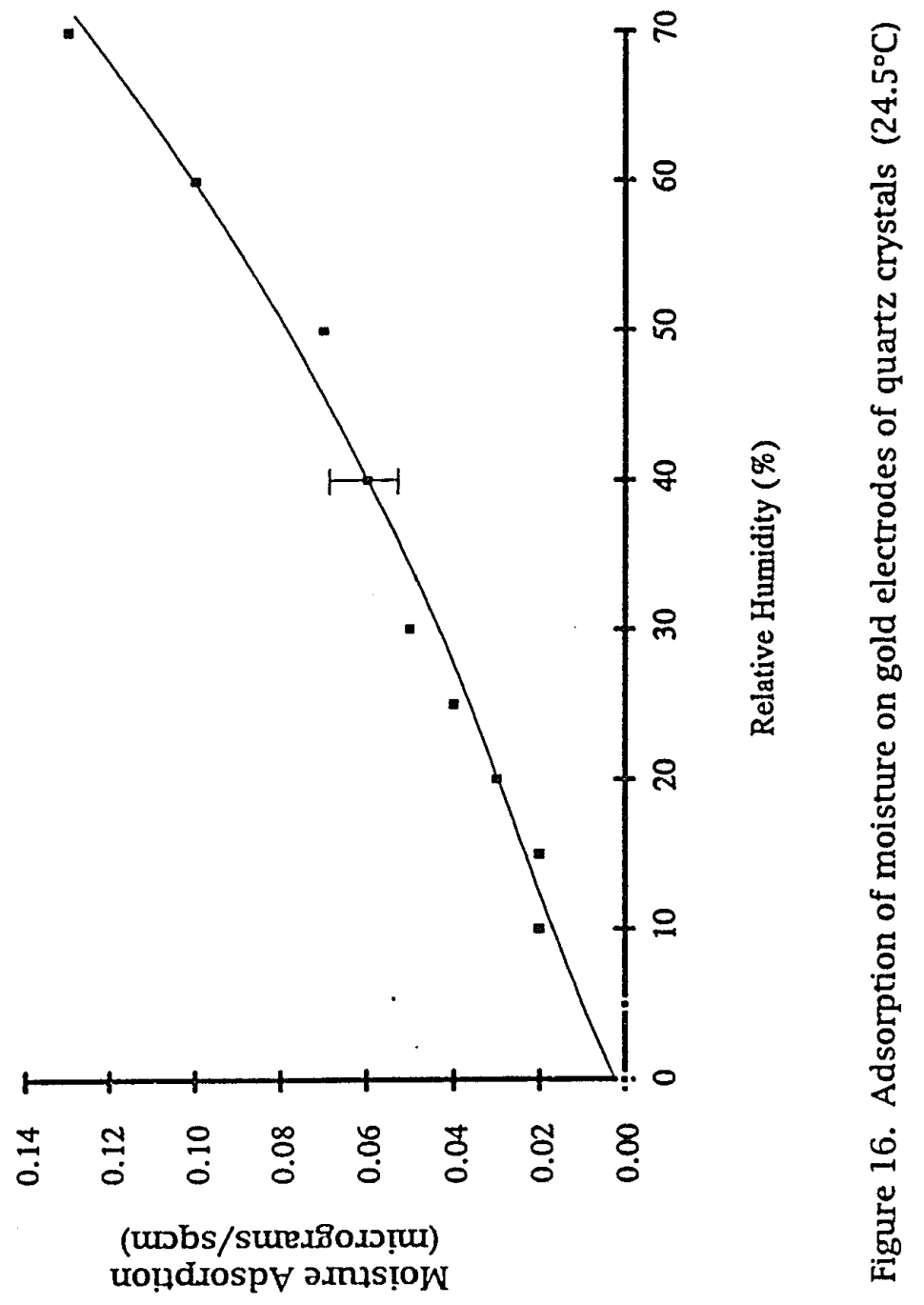
exist. Sharma ${ }^{(53)}$ previously reported a Type II adsorption isotherm for the adsorption of water vapor on electroplated gold. The experiments were performed with a QCM technique.

The plotted mass of adsorbed water in Figure 16 is the result of duplicate measurements with eleven crystals (22 measurements). The error bar shown in Figure 16 is one standard deviation of the variation for all twenty two evaluations and was 0.017 ugrams of water $/ \mathrm{cm}^{2}$ of crystal surface. The standard deviation for multiple runs of a single crystal was less than 0.01 ugrams $/ \mathrm{cm}^{2}$. This was measured from triplicate runs on three crystals.

The $X T C / 2$ QCM is factory programmed to read in angstroms because it is typically used for thickness control in vacuum deposition systems. For this study the weight of adsorbed water was calculated as follows:

$$
M_{a d}=t A_{c} d\left(1 \times 10^{-6}\right)
$$

where

$$
\begin{aligned}
& M_{a d} \quad=\text { adsorbed water }(\mu g r a m s) \\
& t \quad=\text { adsorbed thickness }(\mathrm{cm}) \\
& A_{c} \quad=\text { area of adsorption }\left(2 \times 0.527 \mathrm{~cm}^{2}\right) \\
& d \quad=\text { density of water }(1 \mathrm{~g} / \mathrm{cc})
\end{aligned}
$$

With an assumption of close packing of molecules the mean cross sectional area of a water molecule has been determined to be $0.106 \mathrm{~nm}^{2}$. $^{(54)}$ If one water molecule has a mean cross-sectional area of $0.106 \mathrm{~nm}^{2}$, a $1 \mathrm{~cm}^{2}$ area will have $9 \times 10^{14}$ molecules or $0.028 \mu \mathrm{g}$ of adsorbed water. Since the total active area of the quartz crystal was 1.05 $\mathrm{cm}^{2}$, one complete geometric monolayer would have a mass of $0.031 \mu \mathrm{g}$. Figure 16 shows that a weight of water corresponding to one geometric monolayer is adsorbed, from a $24.5^{\circ} \mathrm{C}$ environment, at approximately $20 \% \mathrm{RH}$. 
In Chapter 2, Figure 6B, it was shown that the Type II isotherm could be interpreted in a quantitative fashion, which allowed the calculation of the monolayer capacity. In order to obtain a proper value for monolayer capacity, the build-up of the monolayer should be virtually complete before the formation of the multilayer commences. This is the clearly identifiable Point B on the Type II isotherm. In systems which give rise to a Type III isotherm the multilayer is being built up on some parts of the surface while the monolayer is still incomplete on others. This is the reason no attempt was made to quantify the isotherm through the use of Equation [7].

To determine if the moisture adsorption was time dependent, the rate of relative humidity increase was varied by increasing the flow through the water bubbler. The fastest humidity ramp that could be obtained between 0 and $70 \% \mathrm{RH}$ was approximately 5 minutes. The amount of water adsorbed was independent of time for isothermal adsorptions ranging from 5 minutes up to 72 hours for RH increasing from 0 to $70 \%$. As has been noted by Adamson, ${ }^{(55)}$ physical adsorption equilibrium is attained very rapidly (typically $<1$ second) except when limited by mass transport rates in the gas phase or within porous solids.

The chamber response for desorption curves was marginally slower, taking 9 minutes for a relative humidity decrease from 70 to $10 \%$ and approximately one hour to decrease from 10 to $0 \% \mathrm{RH}$. Desorption curves were obtained from the fastest possible reduction in $\mathrm{RH}$ and from slow humidity decreases taking up to 72 hours. The desorption curve was also independent of time.

The preliminary studies of moisture adsorption on the gold electrodes of the Leybold-Inficon quartz crystals showed moisture adsorption increasing with RH. It also indicated that the amount of adsorbed moisture was repeatable for the complete 
adsorption process, with a standard deviation of only $0.017 \mu \mathrm{grams}$. The weight of moisture adsorbed was repeatable from crystal to crystal and was independent of time.

\subsection{Moisture adsorption on DC magnetron sputtered carbon}

Figure 17 shows the adsorption isotherms for water vapor adsorbed on DC magnetron sputtered carbon. The carbon had been deposited on the gold electrodes of quartz crystals as described in Section 4.5. The results are averages for 2 crystals with $25 \mathrm{~nm}, 3$ crystals with $50 \mathrm{~nm}$, and 2 crystals with $100 \mathrm{~nm}$ thicknesses of carbon. It is apparent from Figure 17 that the amount of moisture adsorbed increases with carbon thickness. Table 5 shows the results (see Appendix) of a Student t-test calculating the probability that the water vapor adsorption for the four crystal surfaces represented in Figure 17 are significantly different. The results show that the average adsorption, for the crystals with $25 \mathrm{~nm}$ thick carbon, was significantly higher than the adsorption of moisture on the gold electrodes of the quartz crystals. The $50 \mathrm{~nm}$ thick carbon adsorbed more moisture than the $25 \mathrm{~nm}$ thick carbon and the $100 \mathrm{~nm}$ thick carbon adsorbed significantly more moisture than any of the other crystals.

The largest standard deviation $(\sigma)$ for the $70 \%$ RH measurements was $0.06 \mu$ grams $/ \mathrm{cm}^{2}$ and is shown in Figure 17 for the $50 \mathrm{~nm}$ carbon sample. This data point is the largest $\sigma$ of any adsorption point for any carbon thickness. The $\sigma$ represents seven trials for the three $50 \mathrm{~nm}$ thick sputtered carbon films. The $\sigma$ shown for the $70 \% \mathrm{RH}$ adsorption point therefore represents total experimental error including variation in deposition thickness, variation due to the quartz crystal, and all possible variables introduced in the adsorption process.

The adsorption and desorption isotherms shown in Figure 17 exhibit a BET Type II behavior characterized by a distinct slope change at low relative humidity. The slope change was shown in the Type II isotherm described in Section 2.4 and was identified as 


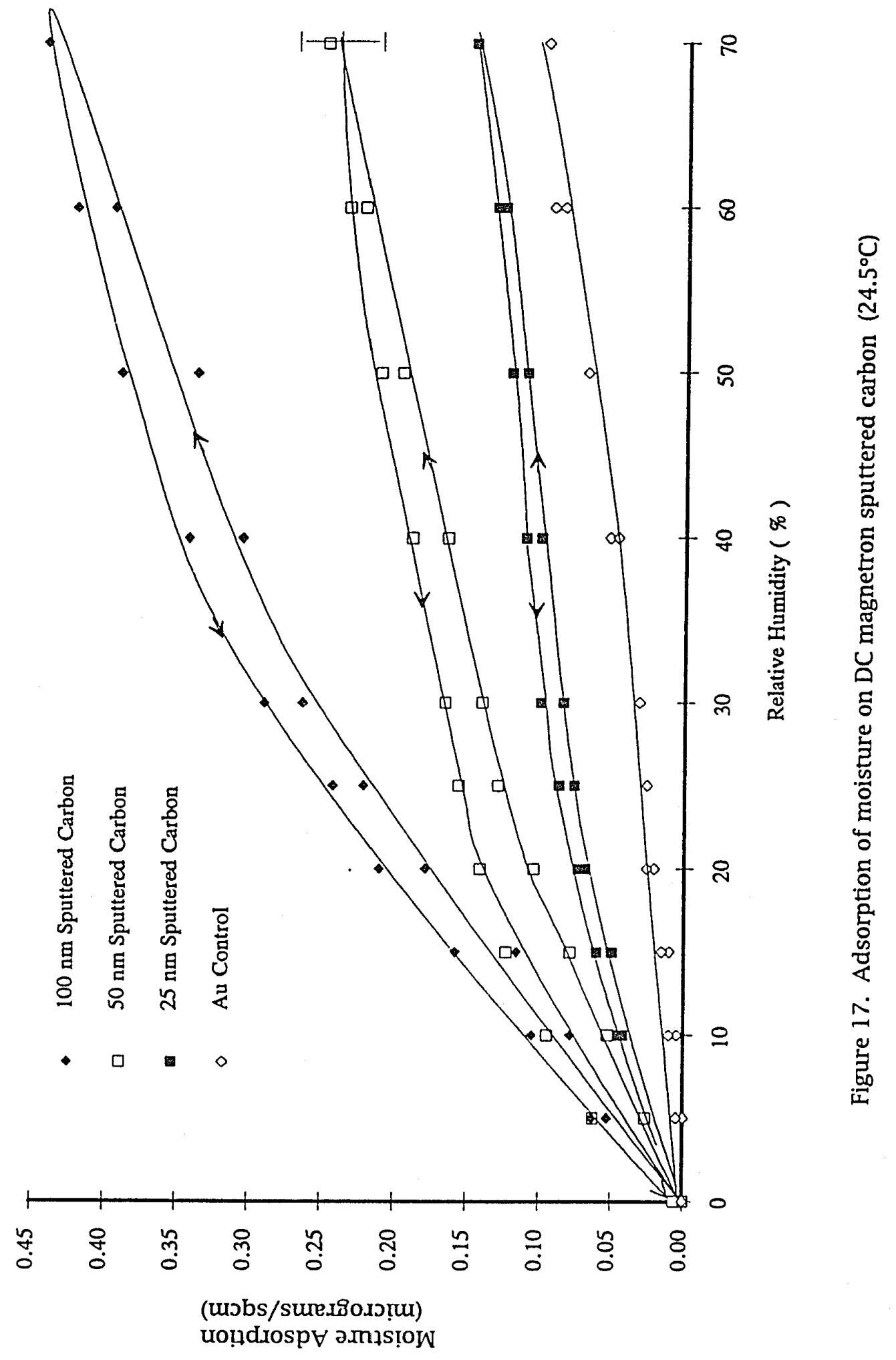


Table 5 - Student $t$-test for significant differences in mass of adsorbed moisture at $70 \%$ relative humidity versus sputtered carbon thickness

\begin{tabular}{lcc}
\hline \multicolumn{1}{c}{ Comparison } & $\begin{array}{c}\text { Mean } \\
(\mu g r a m s)\end{array}$ & $\begin{array}{c}\text { Probability of significant difference in means } \\
\text { Student t-test }\end{array}$ \\
\hline Au vs. $25 \mathrm{~nm}$ carbon & 0.09 vs. 0.13 & 0.93 \\
$25 \mathrm{~nm}$ vs. $50 \mathrm{~nm}$ carbon & 0.13 vs. 0.24 & 0.96 \\
$50 \mathrm{~nm}$ vs. $100 \mathrm{~nm}$ carbon & 0.24 vs. 0.43 & 0.99 \\
\hline
\end{tabular}

Point B. It is typically defined as the breaking point of monolayer and multilayer adsorption. Point B is not well defined for all carbon thicknesses shown in Figure 17 but in general occurs between 20 and $30 \%$ relative humidity.

The quantitative treatment of the BET equation was discussed in Section 2.4. It was shown how the specific surface area $\left(A_{m}\right)$ and heat of adsorption (Equation [7]) could be calculated from the treatment proposed by Brunauer, Emmett, and Teller. Several conditions should be met before an accurate evaluation of specific surface area or heat of adsorption can be obtained. ${ }^{(56)}$ These are:

1) The isotherm must have a sharp knee and a well defined Point $B$.

2) A number of practical requirements must be met: the adsorptive must be chemically inert towards the solid; the saturation vapor pressure $\left(p^{\circ}\right)$ at the working temperature must be large enough to allow accurate measurements of relative pressure over a reasonably wide range $\left(\sim 0.001<p / p^{\circ}<0.5\right)$.

3) Finally, it is desirable that the adsorbate molecule should not be far removed from spherical symmetry to minimize the uncertainty in $A_{m}$ from different possible orientations on the surface.

In a comprehensive survey of the literature, published in 1967 covering 188 references, McClellan and Harnsberger ${ }^{(57)}$ found 128 substances which had been used in 
at least two, and sometimes several surface area evaluations. Despite the large number of materials surveyed, they were able to arrive at recommended values of $A_{m}$ for only five adsorptives. These were nitrogen, argon, krypton, n-butane, and benzene. The conclusion was that water is unsuitable as an adsorptive for the cvaluation of total surface area due to its complexity.

Despite the above arguments to the contrary, it is worth looking at the approximate Point B inflections shown in the carbon isotherms of Figure 17. The weight adsorbed corresponding to Point B can be converted to an approximate surface area by converting the $\mu$ grams adsorbed to number of molecules and multiplying by $0.106 \mathrm{~nm}^{2}$ per molecule. The relative surface area can be defined as the Point $B$ surface area divided by the geometric area of the sputtered carbon. The relative surface area for each carbon thickness is shown in Table 6. These areas show the surface area, as measured by the adsorption of moisture, is increasing in proportion to the carbon thickness. If the area of adsorbed water at $70 \% \mathrm{RH}$ is calculated with the same technique, the area of adsorbed moisture is approximately twice the monolayer capacity. If the thickness of one water molecule is assumed to be $0.3 \mathrm{~nm}$ then two monolayers would be approximately $0.6 \mathrm{~nm}$ thick. As previously mentioned, this assumes that the multilayer does not start until monolayer adsorption is complete.

Table 6. The relative surface area of DC magnetron sputtered carbon as a function of thickness

\begin{tabular}{ccc}
\hline $\begin{array}{c}\text { Carbon Thickness } \\
(\mathrm{nm})\end{array}$ & $\begin{array}{c}\text { Point B Adsorption } \\
\left(\mu \mathrm{g} / \mathrm{cm}^{2)}\right.\end{array}$ & $\begin{array}{c}\text { Relative Surface Area } \\
\left(\mathrm{cm}^{2} / \mathrm{cm}^{2}\right)\end{array}$ \\
\hline 25 & 0.075 & 2.7 \\
50 & 0.12 & 4.3 \\
100 & 0.27 & 9.7 \\
\hline
\end{tabular}


One possible explanation for the increase in moisture adsorption would be an actual increase in specific surface area with increased sputtering time. Atomic force microscope (AFM) micrographs of the surfaces of $25 \mathrm{~nm}$ and $100 \mathrm{~nm}$ carbon thicknesses are shown in Figures 18 through 21 . Figure 18 is a three dimensional AFM section of the $25 \mathrm{~nm}$ thick DC magnetron carbon film. Figure 19 is a three dimensional section of a $100 \mathrm{~nm}$ thick DC magnetron sputtered carbon film. It is obvious that the $100 \mathrm{~nm}$ thick carbon film is substantially rougher. Figure 20 is a line scan of the same $25 \mathrm{~nm}$ thick carbon film and shows an average surface roughness $\left(R_{a}\right)$ of $0.425 \mathrm{~nm}$. Figure 21 shows an AFM line scan of the $100 \mathrm{~nm}$ thick film with an $\mathrm{R}_{\mathrm{a}}$ of $1.18 \mathrm{~nm}$. The increased roughness for the thicker carbon would indicate that more surface area has been created.

Figure 17 also shows a small but significant hysteresis between the adsorption and desorption curves of the carbon surfaces. One explanation for this phenomenon might be capillary condensation in pores. As described by Shaw, ${ }^{(58)}$ the contact angle on adsorption, when liquid is advancing over a dry surface, is generally greater than the contact angle during desorption when liquid is receding from a wet surface. From the Kelvin equation

$$
R T \ln \left(P_{r} / P\right)=-(2 \gamma V \cos \theta) / r
$$

where

$$
\begin{aligned}
& R=\text { universal gas constant } \\
& T=\text { absolute temperature }\left({ }^{\circ} \mathrm{K}\right) \\
& P=\text { vapor pressure of liquid on plane surface } \\
& P_{r}=\text { vapor pressure of liquid droplet (capillary) } \\
& \gamma=\text { liquid surface tension } \\
& V=\text { molar volume of liquid }
\end{aligned}
$$




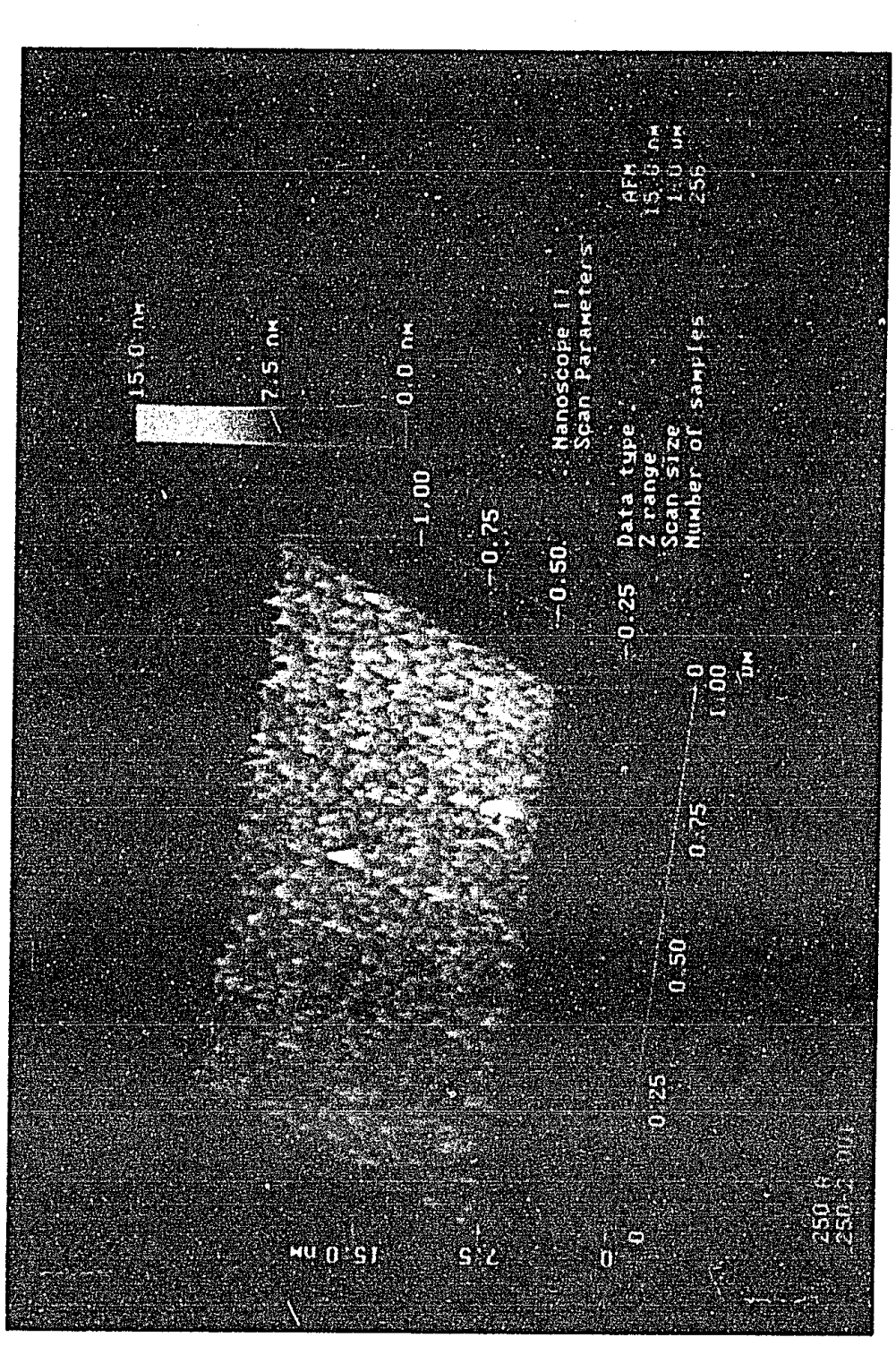

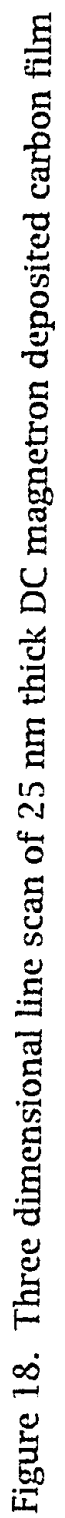




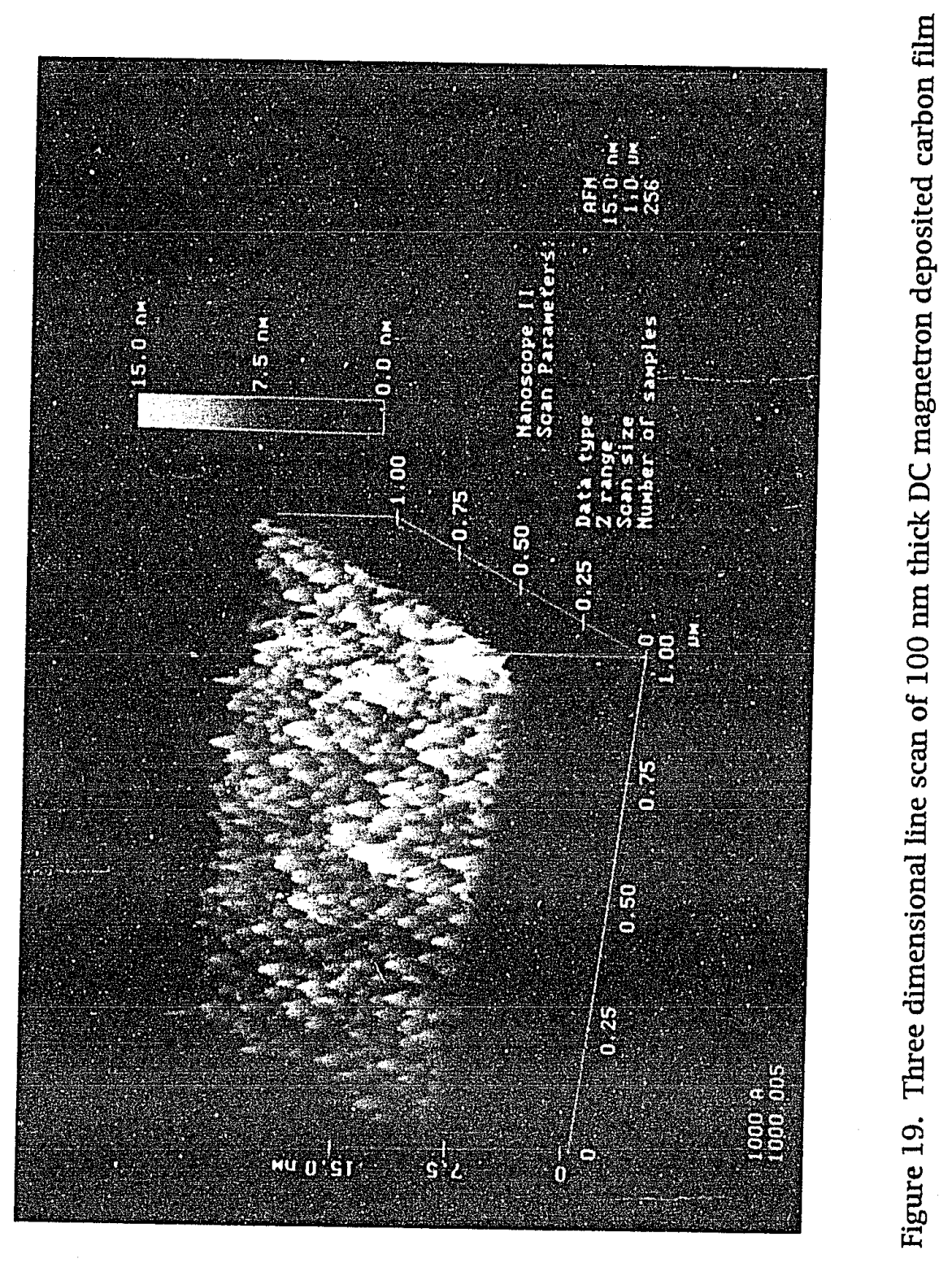




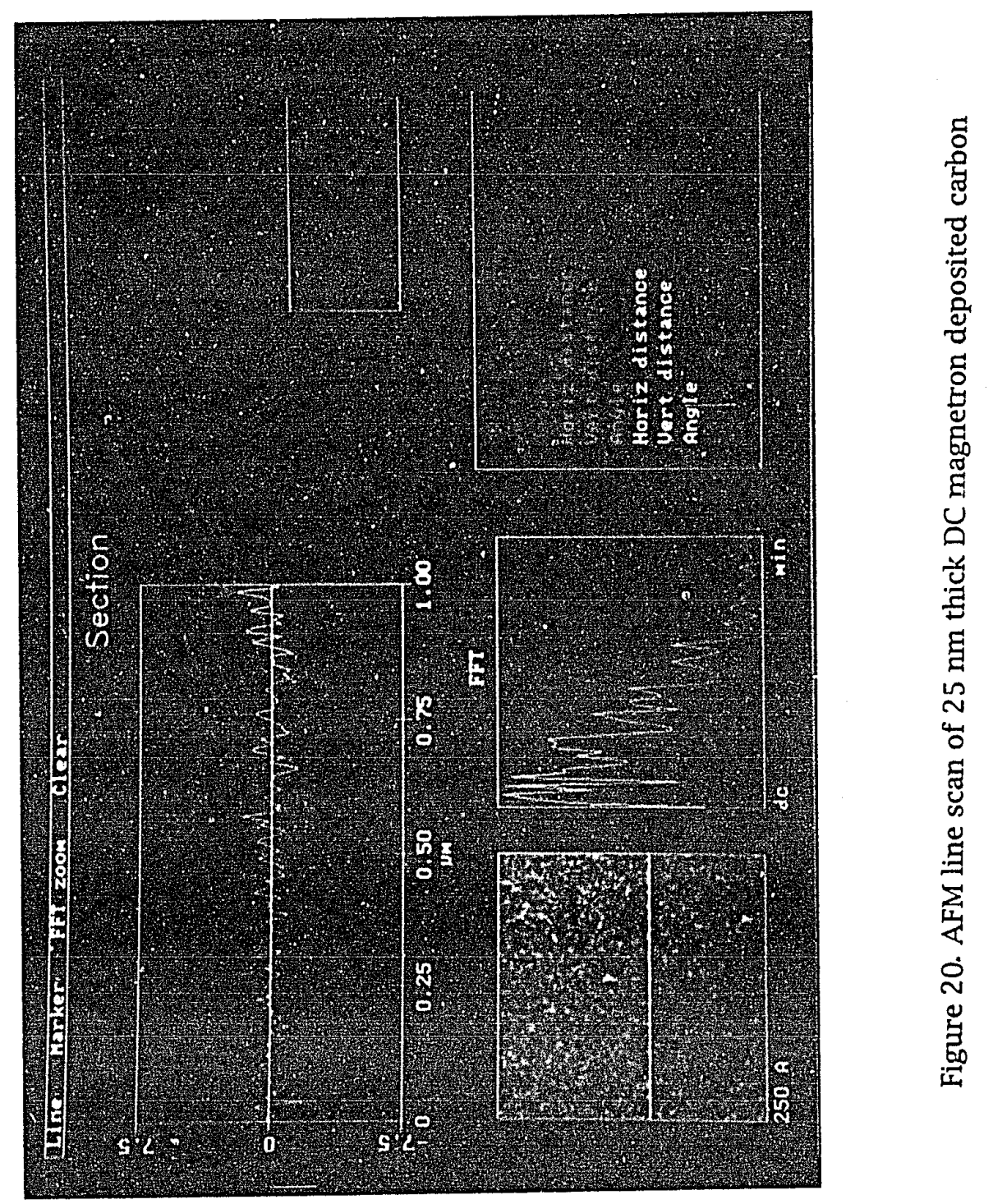




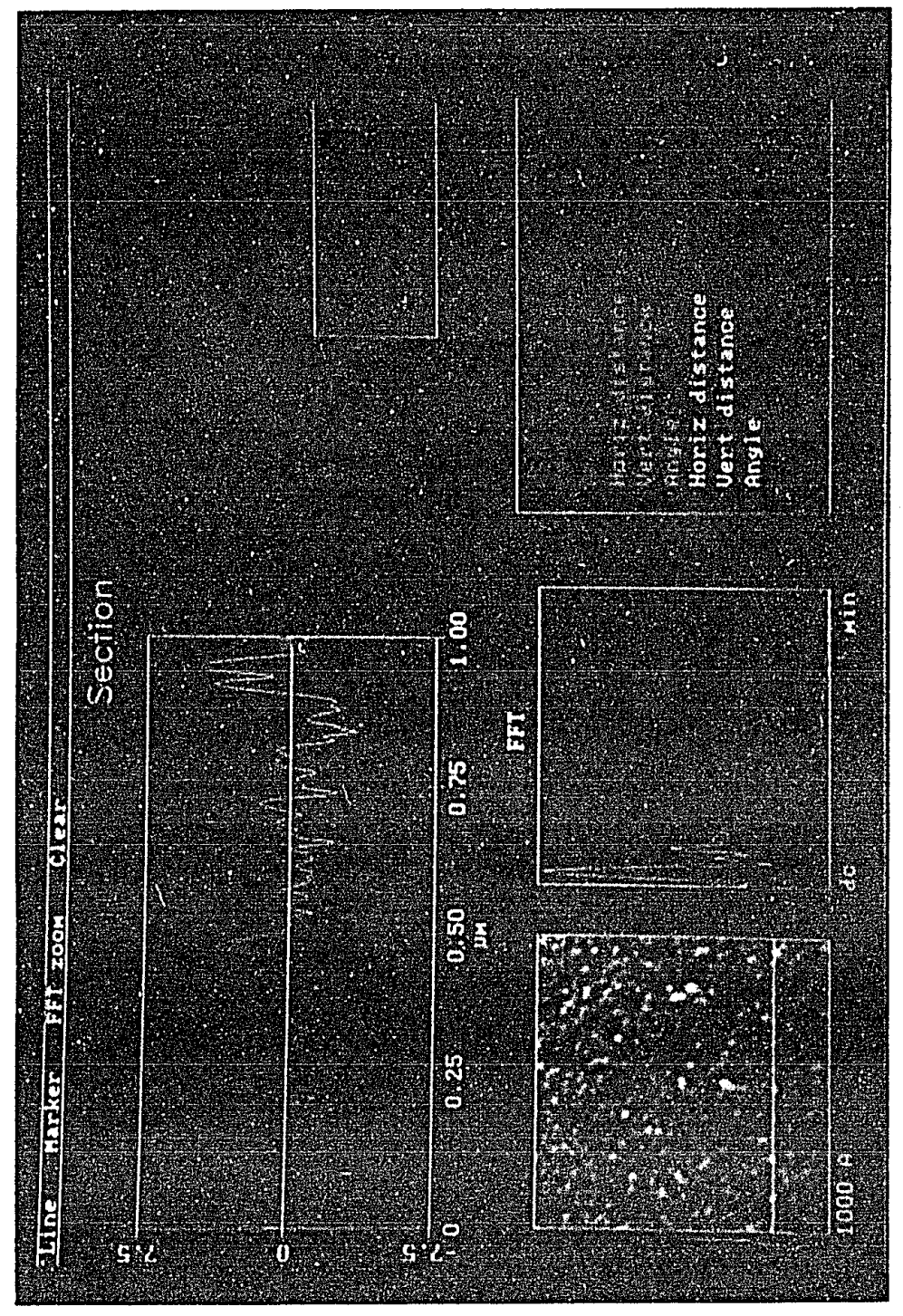

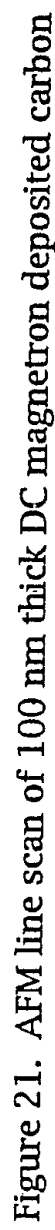




$$
\begin{aligned}
& \theta=\text { contact angle } \\
& r=\text { radius of capillary pore }
\end{aligned}
$$

it is evident that the pressure below which liquid vaporizes from a particular capillary will be lower than the pressure required for capillary condensation. While the above argument is generally accepted as the reason for hysteresis in adsorption isotherms it may not conclusively explain the hysteresis in the moisture adsorption isotherms of this study. It would seem reasonable that an adsorbed monolayer would exist on the pore walls before capillary condensation takes place. If this were true, the hysteresis loop would only be present above a certain relative humidity. An example of this can be seen in the Type $V$ isotherm of Figure 6.

Some literature explanations for low RH hysteresis have been proposed but they remain somewhat speculative. Water is an adsorbate which is particularly prone to show penetration effects due to its small molecular size, its ability to rehydrate or rehydroxylate various oxides and its ability to dissolve many ionic solids. The explanation of low pressure hysteresis proposed by Arnell and McDermott ${ }^{59}$ some thirty years ago was formulated in terms of the swelling of the particle which accompanies adsorption. The swelling distorts the structure and opens up cavities which were previously inaccessible to absorbate molecules. Since the distortion is not perfectly elastic some molecules become trapped and can escape only very slowly, or possibly not at all, during the desorption process. The above mechanism has never been proposed for adsorption on thin films.

As a general summary, the adsorption of moisture on DC magnetron sputtered carbon is dependent on RH. Depending on the roughness of the carbon surface, which was controlled by the deposited thickness, from 0.15 to $0.45 \mu$ grams of moisture $/ \mathrm{cm}^{2}$ of carbon was adsorbed at 70\% RH. The DC magnetron sputtered carbon adsorption 
isotherm has strong characteristics of a BET Type II isotherm with a fairly distinct monolayer capacity slope change in the adsorption isotherm curve. Some hysteresis was present in the adsorption isotherm and may have been caused by porosity or water penetration in the carbon structure. Within the limits of this investigation it was not possible to resolve the hysteresis mechanism and further studies would be necessary to determine if porosity or penetration are reasonable explanations.

\subsection{Adsorption of moisture on DC magnetron sputtered carbon as a function of RH and temperature}

Figure 22 shows the adsorption of moisture on DC magnetron sputtered carbon as a function of $\mathrm{RH}$ at $24.5,29.5$, and $34.5^{\circ} \mathrm{C}$. The results verify that temperature does not affect the weight of moisture adsorbed on DC magnetron sputtered carbon when it is evaluated as a function of RH. Due to time constraints only the adsorption curves were determined. Due to time limitations no experimental data was taken for the decreasing RH (desorption) curves.

The fact that adsorption processes are exothermic was discussed in Section 2.4 (Equation [2]). From thermodynamic relationships the extent of adsorption would increase with decreasing temperature under equilibrium conditions. If the adsorption isotherm in Figure 22 was replotted as the amount of adsorbed water versus the absolute pressure of the water vapor, the higher temperature would show lower adsorption. This is obvious since at an equivalent RH the absolute pressure of water, at a higher temperature, is higher than the absolute pressure at a lower temperature. 


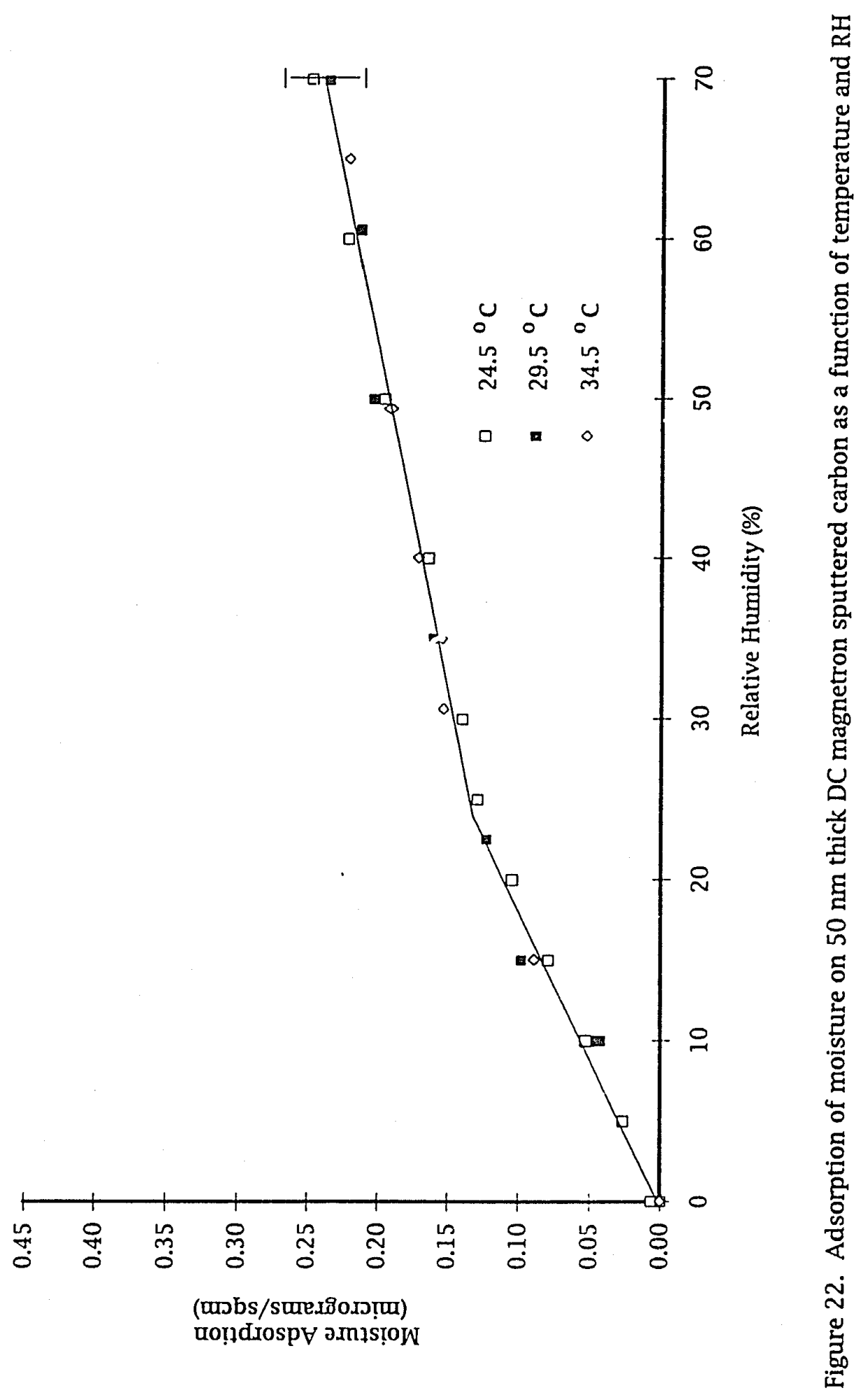




\subsection{Moisture adsorption on PECVD carbon}

Figure 23 shows the adsorption and desorption of water vapor on PECVD deposited carbon films of 50 and $100 \mathrm{~nm}$ thicknesses. The two carbon adsorptions are compared to the adsorption of moisture on the gold electrodes of a quartz crystal. The values shown in Figure 23 are the result of two evaluations with two $50 \mathrm{~nm}$ thick PECVD films and two evaluations with two $100 \mathrm{~nm}$ thick PECVD carbon film.

Figure 23 indicates the weight of water adsorbed on both PECVD carbon thicknesses and the gold control are significantly different. Table 7 shows the results of a Student t-test calculating the probability of a significant difference in mean values. The results show the amount of moisture adsorbed increased with increasing thickness of PECVD carbon and both carbon films adsorbed more moisture than the gold electrode of the quartz crystal.

Table 7 - Student t-test for significant differences in amount of moisture adsorption at $70 \%$ relative humidity versus thickness of PECVD carbon

\begin{tabular}{llc}
\hline Comparison & $\begin{array}{l}\text { Mean } \\
\text { ( } \mu \text { grams) }\end{array}$ & $\begin{array}{c}\text { Probability of significant difference in means } \\
\text { Student t-test }\end{array}$ \\
\hline Au vs. $50 \mathrm{~nm}$ PECVD carbon. & 0.09 vs. 0.23 & 0.99 \\
50 vs. $100 \mathrm{~nm}$ PECVD carbon. & 0.23 vs. 0.47 & 0.99 \\
\hline
\end{tabular}

The relative surface area can be calculated for the PECVD carbon in the same way it was calculated for the DC magnetron deposited carbon. The results of these calculations, shown in Table 8 , indicate that moisture adsorption increases with carbon thickness approximately in proportion to the increase in relative surface area.

As in the case of DC magnetron sputtered carbon the area of water adsorbed at $70 \%$ RH is approximately twice the monolayer capacity. Ignoring the competition 


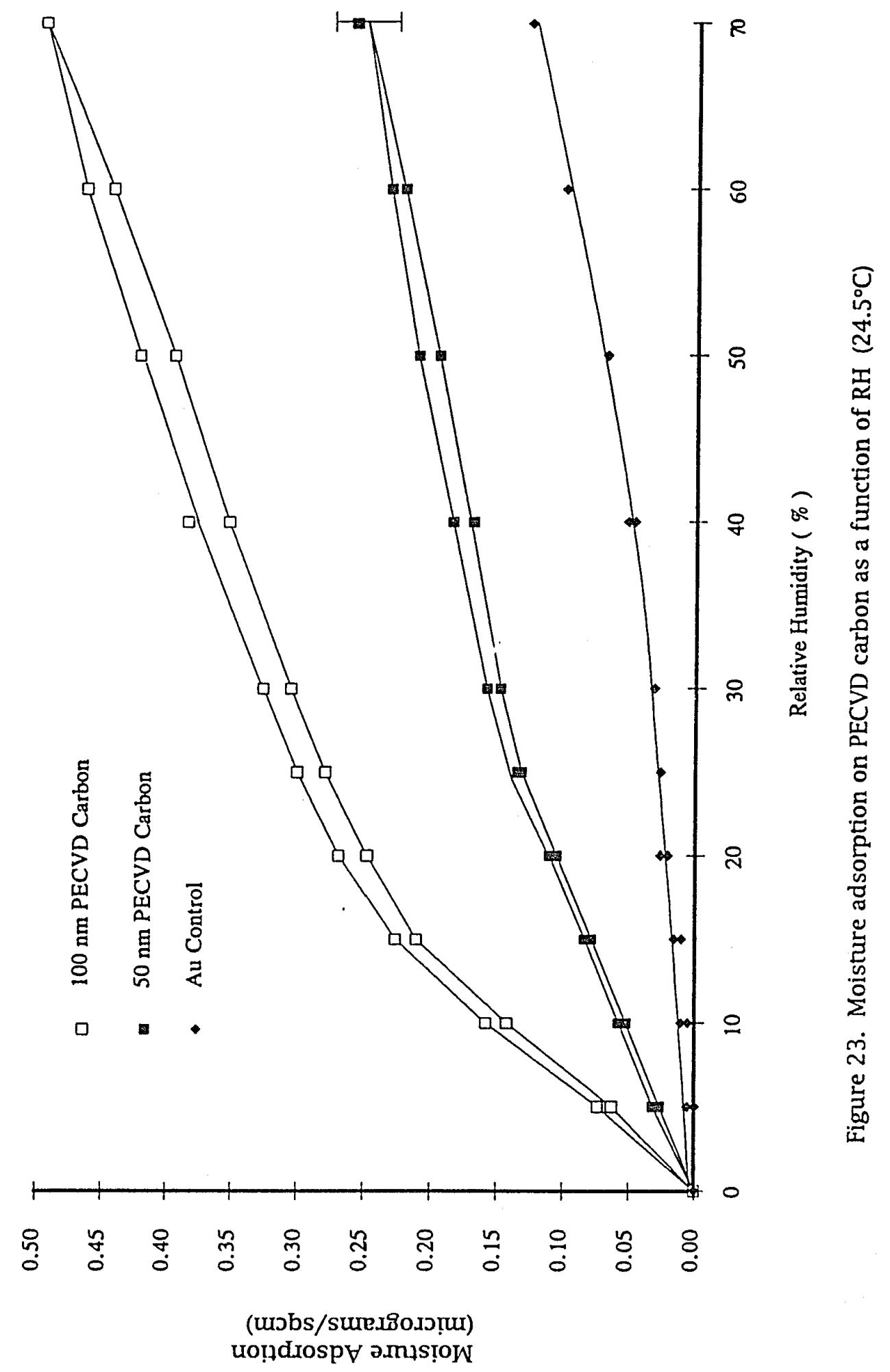


between monolayer and multilayer adsorption approximately of $0.6 \mathrm{~nm}$ of water is adsorbed at $70 \% \mathrm{RH}$.

As in the case of DC magnetron sputtered carbon, a small but significant hysteresis is shown for the moisture adsorption and desorption curves of the PECVD

Table 8 - The relative surface area of PECVD carbon as a function of thickness

\begin{tabular}{ccc}
\hline $\begin{array}{c}\text { Carbon Thickness } \\
(\mathrm{nm})\end{array}$ & $\begin{array}{c}\text { Point B adsorption } \\
\left(\mu \mathrm{g} / \mathrm{cm}^{2}\right)\end{array}$ & $\begin{array}{c}\text { Relative Surface Area } \\
\left(\mathrm{cm}^{2} / \mathrm{cm}^{2}\right)\end{array}$ \\
\hline 50 & 0.13 & 4.7 \\
100 & 0.23 & 8.2 \\
\hline
\end{tabular}

carbon. The adsorption and desorption isotherms shown in Figure 23 also exhibit a BET Type II behavior with a distinct slope change at low relative humidity. This point is fairly well defined for both PECVD carbon thicknesses and occurs between 15 and 30\% relative humidity.

Figure 24 is an AFM line scan of a $50 \mathrm{~nm}$ thick PECVD carbon film and Figure 25 is an AFM line scan of a $100 \mathrm{~nm}$ thick PECVD carbon film. The $50 \mathrm{~nm}$ film shown in Figure 24 has a roughness $\left(\mathrm{R}_{\mathrm{a}}\right)$ of $0.09 \mathrm{~nm}$ while the $100 \mathrm{~nm}$ film shown in Figure 25 has an $R_{a}$ of $0.13 \mathrm{~nm}$. As with the $D C$ magnetron sputtered carbon, the thicker film is also rougher.

The adsorption studies with PECVD carbon exhibited similarities to the DC magnetron carbon. The moisture adsorption increased with carbon thickness and a slight hysteresis in the adsorption isotherm was found. The thicker PECVD carbon was rougher which probably accounted for its increased moisture adsorption. The relative surface area of the PECVD carbon, as measured by the adsorption of moisture, was within $18 \%$ of the relative surface area of the DC magnetron sputtered carbon. 


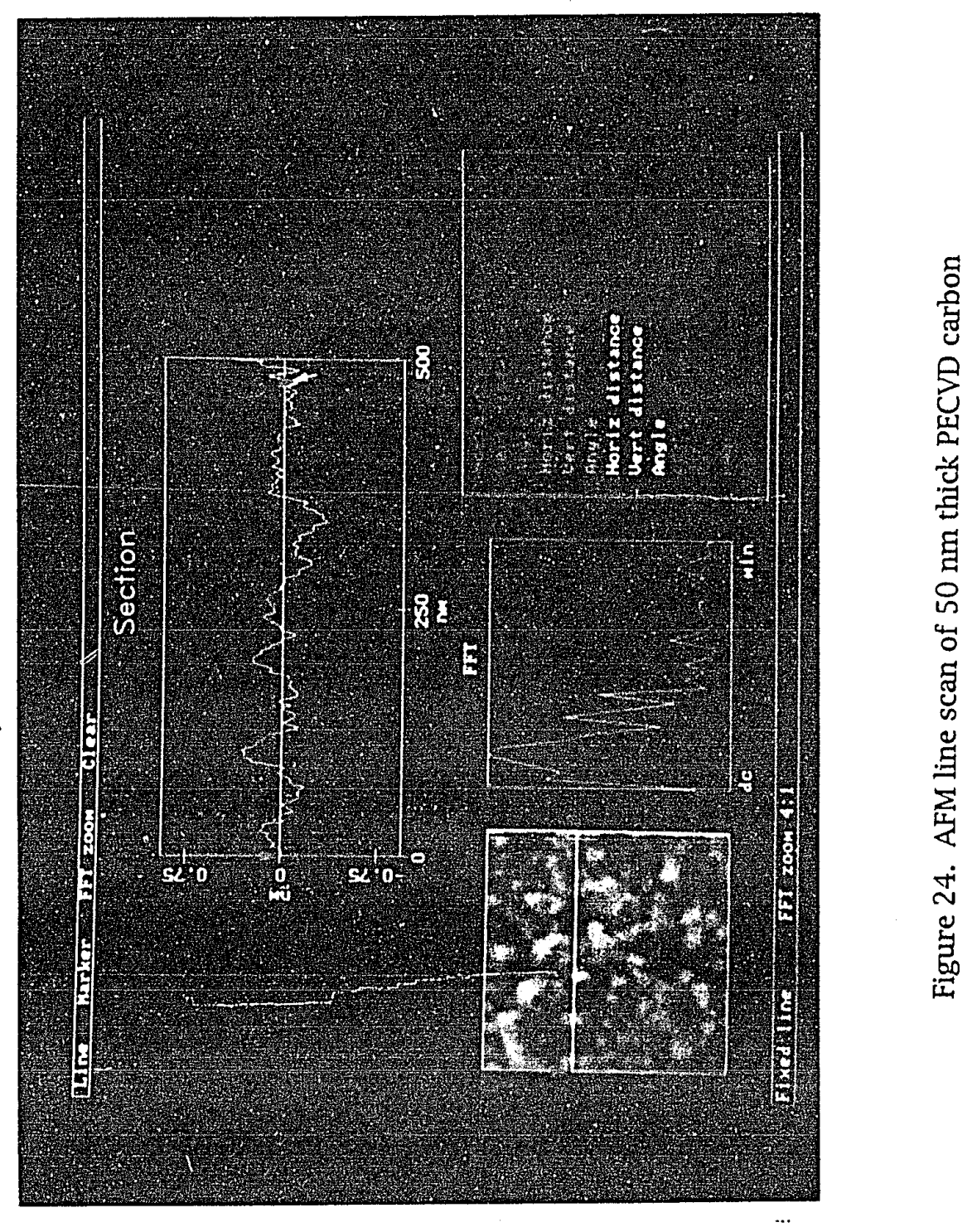




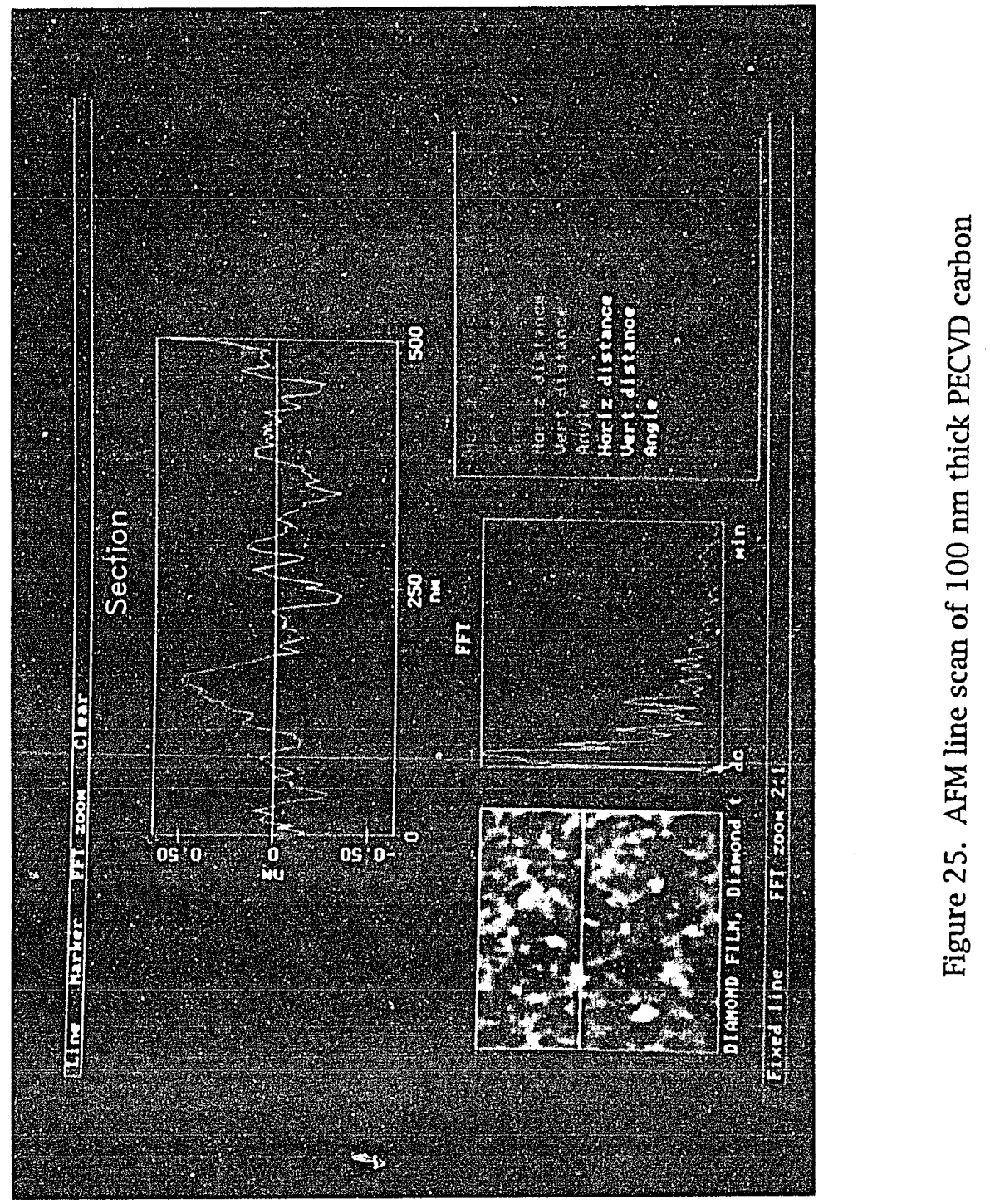




\subsection{Comparison of moisture adsorption on PECVD and DC magnetron sputtered carbon films}

Figure 26 plots the adsorption of moisture on DC magnetron sputtered carbon and on PECVD carbon as a function of relative humidity. It has become obvious from the previous two sections that the amount of adsorbed moisture did not vary significantly between the PECVD and DC magnetron sputtered carbon. A Student t-test confirmed a low probability $(<0.8)$ that the weight of water vapor adsorbed on PECVD deposited carbon was different than the amount adsorbed by DC magnetron sputtered carbon when the thickness is held constant.

In a recent publication Tian and Matsudaira ${ }^{(60)}$ found that an unlubricated carbon overcoated magnetic disk adsorbed $0.3 \mathrm{~nm}$ of moisture at $50 \% \mathrm{RH}, 0.6 \mathrm{~nm}$ at $80 \% \mathrm{RH}$ and $0.9 \mathrm{~nm}$ at $90 \% \mathrm{RH}$. The measurements were made with an ellipsometer in an environmental chamber. No indication of the carbon thickness is given but the surface roughness $\left(R_{a}\right)$ was measured by a profilometer technique as $0.64 \mathrm{~nm}$. In an earlier publication Li, Trauner and Talke ${ }^{(61)}$ found that a lubricated carbon overcoated disk adsorbed 0.4 to $0.6 \mathrm{~nm}$ of moisture between 60 and $80 \% \mathrm{RH}$. Their technique again used an ellipsometer in an environmental chamber.

In this study the calculations for relative surface area and adsorption at $70 \% \mathrm{RH}$ indicate two molecular layers of water at $70 \% \mathrm{RH}$. As previously shown, this can be converted to $0.6 \mathrm{~nm}$ of water by assuming a water molecule thickness of $0.3 \mathrm{~nm}$. This is in excellent agreement with the two published studies on moisture adsorption. 


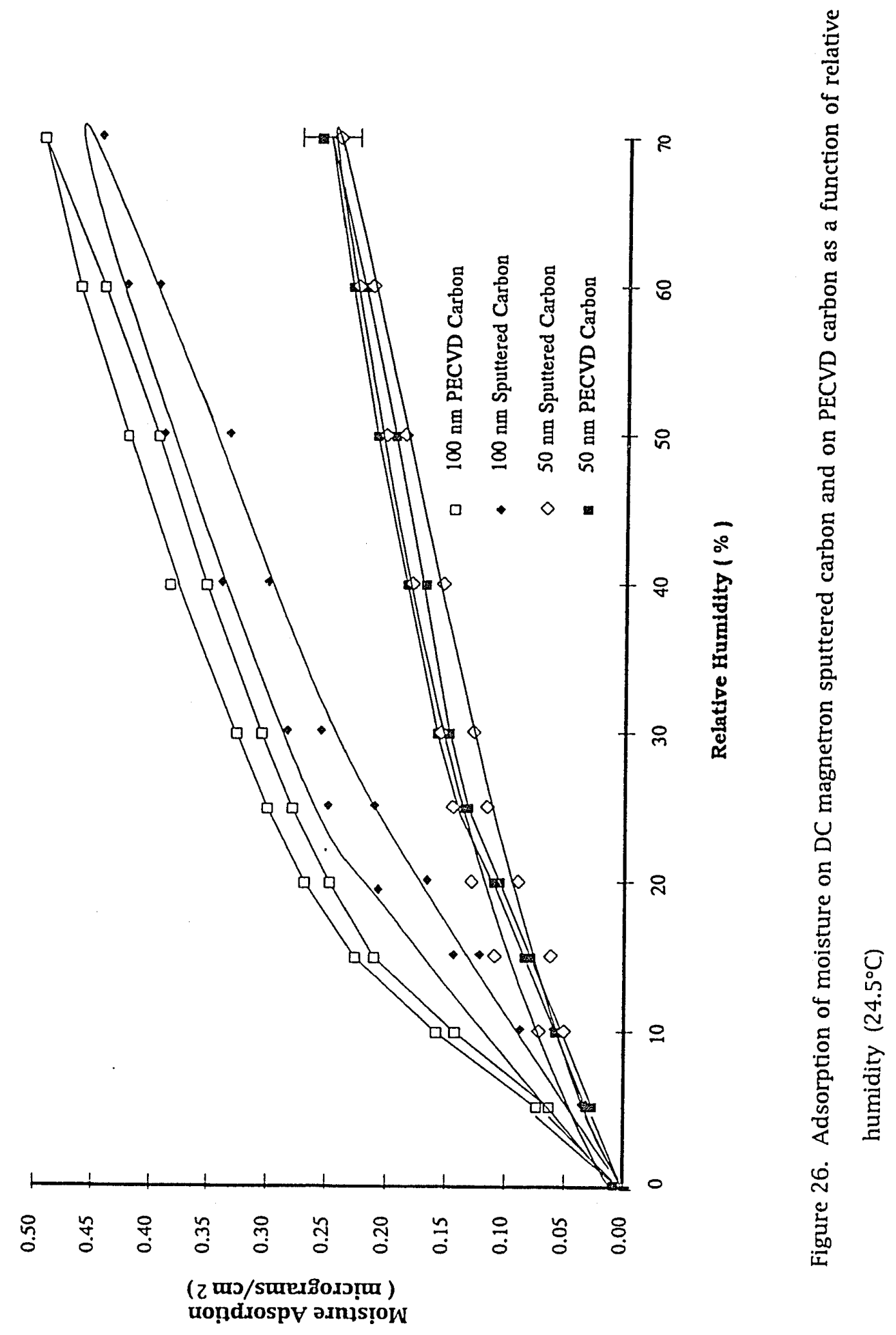




\section{CHAPTER 6}

\section{Conclusion}

This study has detailed the development of a microbalance technique capable of evaluating the adsorption of moisture on thin carbon films used as overcoats for magnetic rigid disks. The apparatus was a simple aluminum chamber with temperature and humidity controls, relative humidity and temperature monitoring equipment, and a Leybold-Inficon quartz crystal microbalance.

The study of moisture adsorption using this technique has shown that the weight of moisture adsorbed on carbon thin films depends on the roughness of the carbon surface and the RH. The amount of moisture adsorbed at equal carbon thickness did not vary significantly between carbon deposited by plasma enhanced chemical vapor deposition and DC magnetron sputtering. The hydrogen content, hardness, and resistivity of the two carbons were significantly different. The amount of moisture adsorbed on both carbons was significantly different than the amount of moisture adsorbed on the gold electrodes of the quartz crystals. The total experimental error of the quartz crystal microbalance technique was $0.06 \mu \mathrm{g}$ of moisture $/ \mathrm{cm}^{2}$ of carbon surface.

This technique showed that approximately two monolayers of moisture were adsorbed at $70 \% \mathrm{RH}$ for both carbon films. This would correspond to a $0.6 \mathrm{~nm}$ thick film of water. This value was in good agreement with two literature values for adsorbed moisture, at comparable $\mathrm{RH}$, determined using spectroscopic ellipsometry. 


\section{CHAPTER 7}

\section{Recommendations For Future Research}

The following studies are recommended to further improve the understanding of moisture adsorption on carbon thin films:

1) Vary the deposition parameters for DC magnetron sputtering and PECVD carbon to produce equal surface roughness and hopefully maintain the large differences in hardness, resistivity, and hydrogen content. Perform a complete series of adsorption isotherms to allow the development of an equation predicting the amount of moisture adsorbed as a function of the AFM roughness and $\mathrm{RH}$.

2) Construct a quartz crystal microbalance in a vacuum deposition system for the investigation of moisture adsorption before the carbon thin films are exposed to ambient conditions. It is possible that chemical surface differences are masked by the prior adsorption of atmospheric contaminants.

3) Construction of an environmental chamber with improved heating capabilities would allow for a wider range of temperature studies. Hysteresis mechanisms such as moisture penetration are possibly sensitive to the temperature of the adsorption isotherm.

4) The measurement of moisture adsorption by an ellipsometer and quartz crystal microbalance in the same experiment could determine the accuracy and limitations of each technique.

Investigation into the above listed areas should provide insight into the fundamental nature of the adsorption of moisture on carbon thin films. This might 
lead to improved understanding of friction stiction and wear mechanisms of magnetic rigid disks. 


\section{CHAPTER 8}

\section{Endnotes}

1 "1991 Disk/Trend Report," 1992 IDEMA Data Storage Industry Assessment, International Disk Drive Equipment and Materials Association, Sunnyvale, CA (1992).

2 ibid.

3 M. L. Lesser and J. W. Haanstra, "The Random Access Memory Accounting Machine," IBM Journal of Research and Development 1 (1957): 62.

4 C. D. Mee and E. D. Daniel, Magnetic Recording (New York: McGraw-Hill Book Company, 1987), 52.

5 T. C. Arnouldussen and E. M. Rossi, "Materials for Magnetic Recording," Annual Review of Material Science 15 (1985): 379.

6 C. D. Mee and E. D. Daniel, op. cit.

7 Finn Jorgensen, The Complete Handbook of Magnetic Recording (Blue Ridge Summit, PA: Tab Books, 1980), 179.

8 F. E. Talke and R. C Tseng, "An Experimental Investigation of the Effect of Medium Thickness and Transducer Spacing on the Read-Back Signal in Magnetic Recording Systems," IEEE Transactions on Magnetics 9 (1973): 133.

9 S. Tanabe and T. Ozaki, "A Comparison of Perpendicular and Longitudinal Recording for Rigid Disks," Proceedings, Intermag Conference April (1987).

10 R. S. Timsit and G. Stratford, "Effect of Humidity on Friction at Hard Disk Interfaces," Tribology and Mechanics of Magnetic Storage Systems 5 SP-25 (1987): $17-23$.

11 D. W. Rice, P. B. P. Phipps and R. Tremoureux, "Atmospheric Corrosion of Cobalt," Journal of the Electrochemical Society 126 (1979): 1459.

12 F. A. Cotton and G. Wilkinson, Advanced Inorganic Chemistry (New York: Wiley Interscience, 1980), 356.

13 C. Leu, "The Preparation, Characterization, and Tribological Study of RF Sputtered Thin Films for Magnetic Memory Disks," Ph.D. thesis, University of Minnesota (1988). 
$14 \mathrm{H}$. Tsai and D. Bogy, "Characterization of Diamond-like Carbon Films and Their Applications as Overcoats on Thin Film Media for Magnetic Recording," Journal of Vacuum Science and Technology 5 (1987): 6.

15 P. Koidl, "Critical Assessment of Diamond-like Carbon," Proceeding of the First International Symposium of Diamond and Diamond-Like Films, The Electrochemical Society, (1989): 237.

$16 \mathrm{H}$. Tsai and D. Bogy, loc. cit.

17 H. Dimigen and H. Hubsch, "Applying Low Friction Wear Resistant Thin Solid Films by Physical Vapor Deposition," Philips Technical Reviews 41 (1983/84): 186.

18 Timsit and Stratford, loc. cit.

19 E. Rabinowicz, Friction and Wear of Materials (New York: Wiley, 1965), 56.

20 H. Hamilton, R. Anderson and K. Goodson, "Contact Perpendicular Recording with Integrated Head/Flexure," IEEE Transactions on Magnetics 27 (1991): 492.

21 R. D. Fisher, "A Review of Corrosion Testing and Evaluation of Thin-Film Media," Proceedings of IIST Symposium, Report WS\#-B-2 (1986): 1-25.

22 Jeh-Beck Ju and W. H. Smyrl, "Corrosion Studies of Thin Film Materials for Magnetic and Microelectronic Applications," Proceedings of 3rd ASM Conference on Electronic Packaging, (1987): 119-130.

23 J. S. Judge, J. R. Morrison, D. E. Speliotis and G. Bate, "Magnetic Properties and Corrosion Behavior of Thin Electroless Co-P Deposits," Journal of the Electrochemical Society 112 (1965): 681.

24 R. D. Cormia, "Elevated Temperature/Humidity Corrosion of Cobalt Phosphide Plated Magnetic Media," National Association of Corrosion Engineers, Fourth Conference Proceedings, (April, 1990).

25 M. C. Garrison, "Effects of Adsorbed Films on Galvanic Corrosion In Metallic Thin Film Media," IEEE Transactions on Magnetics 19 (1983): 1683.

26 M. Yanagisawa, M. Shiota, H. Yamaguchi and Y. Suganuma, "Corrosion Resisting CoPt Thin Film Medium for High Density Recording," IEEE Transactions on Magnetics 19 (1983): 1737.

27 G. L. McIntire, C. F. Brucker, "Cobalt Chromium Magnetic Recording Media: A Corrosion Study," IEEE Transactions on Magnetics 24 (1988): 2221.

28 R. Dubin, K. Winn, L. Davis and R. Cutler, "Degradation of Co-Based Thin Film Recording Materials In Selected Corrosive Environments," Journal of Applied Physics 53 (1982): 2579. 
29 H. Suzuki, Y. Shiroishi, S. Hishiyama, T. Ohno, Y. Matsuda, F. Matsunaga and N. Tsumita, "Magnetic Properties and Corrosion Resistance of Sputtered $\mathrm{Co}-\mathrm{Ni}$ and $\mathrm{Co}$ Ni-M Films for Longitudinal Recording," IEEE Transactions on Magnetics 23 (1987): 3411 .

30 D. W. Rice, B. Phipps and R. Tremoureux, loc. cit.

31 V. Novotny, G. Itynre, A. Homola and L. Franco, "Corrosion of Thin Film Cobalt Based Magnetic Recording Media," IEEE Transactions on Magnetics 23 (1987): 3645.

32 S. J. Gregg and K. S. Sing, Adsorption, Surface Area and Porosity (London: Academic Press, 1982), 5.

3.3 ibid., 4 .

34 S. Brunauer, P. H. Emmett and E. Teller, "Adsorption of Gases in Nultimolecular Layers," Journal of the American Chemical Society 60 (1938): 309.

35 S. J. Gregg, op. cit., 42.

36 I. Langmuir, "The Constitution and Fundamental Properties of Solids and Liquids," Journal of the American Chemical Society 40 (1916): 2221.

37 M. Ward and D. Buttry, "In Situ Mass Detection with Piezoelectric Transducers," Science $24 !)(190)(0): 1000$.

38 J. F. Nye, Physical Properties of Crystals: Their Representation by Tensors and Matrices, (London: Oxford University Press, 1985), 127.

39) G. Sauerbrey, "Verwendung von Schwingquarzen zur Wägung dünner Schichten und cur Nikrowägung," Zcitschrift für Physik 155 (1959): $20(\mathrm{i}$.

4) J. Niller and D. Bolef, "Acoustic Wave Analysis of Quartz Crystal Film Nonitor," Journal of tpplied Phusics 3!) (1!)(68): 5815.

+1 H. L. Eschbach, and E. W. Kruidhof, "A Direct Calibration Nerhod for a Crystal Oscillator Film Thickness Monitor," Lacuum Mlicro Balance Techniques j (1966): 207.

42 H. K. Pulker, "Progress in Monitoring Thin Film Thickness with Quartz Crystal Resonators," Thin Solid Films 32 (1976): 27.

43 A. W. Warner and C. D. Stockbridge, "Mass Measurement with Crystalline Quartz," Vacuum Micro Balance Techniques 3 (1966): 55.

44 L. J. Slutsky and W. H. Wade, "Adsorption of Gases on Quartz Single Crystals," Journal of Chemical Physics 36 (1962): 2688. 
45 G. M. Kahn, "High Sensitivity Piezogravimetric Method for the Study of Physical Adsorption," Review of Scientific Instruments 43 (1973): 117.

46 J. H. Thomas and S. P. Sharma, "Adsorption and Desorption of Water on Au by the Quartz-Crystal-Oscillator Method," Iournal of Vacuum Science and Technology 13 (1976): 549.

47 J. H. Thomas, and S. P. Sharma, "Adsorption of Water Vapor on Thin-Gold Electroplate on Copper," Journal of Vacuum Science and Technology 14 (1977): 825.

48 D. Rice, P. Phipps and R. Tremoureux, "Atmospheric Corrosion of Cobalt," Journal of the Electrochemical Society 126 (1979): 1459.

49 D. R. Zrudsky, H. D. Bush, and J. R. Fassett, "Four Point Probe Measurement of Surface Conductivity," Review of Scientific Instruments 37 (1966): 885.

50 B. Bhushan, A. Kellock, N. Cho, and J. Ager III, "Characterization of Chemical Bonding and Physical Characteristics of Diamond-like Amorphous Carbon and Diamond Films," Journal of Materials Research 7 (1992): 1459.

51 S. J. Gregg and K.S. Sing, op. cit., 249.

52 ibid.

53 J. H. Thomas and S. P. Sharma, loc. cit.

54 S. J. Gregg and K.S. Sing, op. cit., 238.

55 Arthur W. Adamson, Physical Chemistry of Surfaces 5th ed. (New York: John Wiley, 1990), 593.

56 J. Gregg and K.S. Sing, op. cit., 73.

57 A. L. McClellan and H. F. Harnsberger, "A Review of Gas Solid Adsorption," Journal of Colloid and Interface Science 23 (1967): 577.

58 D. J. Shaw, Introduction to Colloid and Surface Chemistry (London: Butterworths, 1968), 50.

59 J. Gregg and K.S. Sing, op. cit., 235.

$60 \mathrm{H}$. Tian, T. Matsudaira, "Effect of Relative Humidity on Friction Behavior of the Head/Disk Interface," IEEE Transactions on Magnetics 28 (1992): 2530.

61 Y. Li, D. Trauner and F. Talke, "Effect of Humidity on Stiction of the Head/Disk Interface," IEEE Transactions on Magnetics 26 (1990): 2487. 
APPENDIX 
Ver:4 Jul 88

Rapid Data

San Jose CA
MEAN AND VARIANCE TEST

Gold versus $25 \mathrm{~nm}$ DC Magnetron Carbon

Test for difference in moisture adsorption at $70 \% \mathrm{RH}$

DESCRIPTION: SAMPLES

\begin{tabular}{|lrr|l|}
\cline { 2 - 3 } \multicolumn{1}{c|}{} & Gold & 25 nm thick & \\
\hline DATA POINTS & 22.00 & 4.00 & ENTER THE NUMBR OF DATA POINTS \\
MEAN $\left(u g / \mathrm{cm}^{\wedge} 2\right)$ & 0.09 & 0.13 & ENTER THE MEAN \\
STD DEV $(\mathrm{N})$ & 0.016 & 0.040 & ENTER THE STANDARD DEVIATION \\
\hline
\end{tabular}

$$
\begin{aligned}
& T= \\
& D F= \\
& \text { PROB }(-I N F, T)= \\
& \text { PROB }(-T, T)= \\
& F= \\
& D F(N 1, N 2)= \\
& \operatorname{PROB}(0, F)=
\end{aligned}
$$

INSTRUCTIONS

1)

2)
$1.71 \mathrm{~T}$ TEST FOR MEANS. IF $\mathrm{T}<1$, THEN USE A TABLE

3.00 DEGREES OF FREEDOM

0.91 PROB OF A SIG DIF IN MEANS (ONE TAIL TEST)

0.81 PROB OF A SIG DIF IN MEANS (TWO TAIL TEST)

6.25 F TEST FOR VARIANCES

3.00

21.00 DEGREES OF FREEDOM

1.00 PROB OF A SIG DIF IN VARIANCES
ENTER THE DESCRIPTION IN THE CORRECT ORDER. FOR EXAMPLE: PROCESS, \#1 vS \#2. THEN THE \#1 STATISTICS GO UNDER SAMPLE \#1 AND THE \#2 STATISTICS UNDER SAMPLE \#2.

THE T TEST ASSUMES ONLY THAT THE TWO SAMPLES ARE NORMALLY DISTRIBUTED. WE DO NOT ASSUME THAT THE VARIANCES ARE EQUAL. THIS TEST IS MORE GENERAL THAN THE USUAL TEST WHICH ASSUMES EQUAL VARIANCES. 
Ver:4 Jul 88

Rapid Data

San Jose CA

DESCRIPTION:

\begin{tabular}{|lrr|l}
\cline { 2 - 3 } \multicolumn{1}{c|}{} & $25 \mathrm{~nm}$ thick & $50 \mathrm{~nm}$ thick & \\
\hline DATA POINTS & 4.00 & 7.00 & ENTER THE NUMBR OF DATA POINTS \\
MEAN (ug/cm^2) & 0.13 & 0.24 & ENTER THE MEAN \\
STD DEV (N) & 0.060 & 0.060 & ENTER THE STANDARD DEVIATION
\end{tabular}

$\mathrm{T}=$

$\mathrm{DF}=$

PROB $(-$ INF, T $)=$

$\operatorname{PROB}(-T, T)=$

$\mathrm{F}=$

$\mathrm{DF}(\mathrm{N} 1, \mathrm{~N} 2)=$

$\operatorname{PROB}(0, \mathrm{~F})=$

INSTRUCTIONS

1)

2)
MEAN AND VARIANCE TEST

$50 \mathrm{~nm}$ versus $25 \mathrm{~nm}$ DC Magnetron Carbon

Test for difference in moisture adsorption at $70 \% \mathrm{RH}$

SAMPLES

0.060

0.060 ENTER THE STANDARD DEVIATION

2.59 T TEST FOR MEANS. IF T<1, THEN USE A TABLE

6.00 DEGREES OF FREEDOM

0.98 PROB OF A SIG DIF IN MEANS (ONE TAIL TEST)

0.96 PROB OF A SIG DIF IN MEANS (TWO TAIL TEST)

1.00 F TEST FOR VARIANCES

6.00

3.00 DEGREES OF FREEDOM

0.54 PROB OF A SIG DIF IN VARIANCES

ENTER THE DESCRIPTION IN THE CORRECT ORDER.

FOR EXAMPLE: PROCESS, \#1 vS \#2.

THEN THE \#1 STATISTICS GO UNDER SAMPLE \#1

AND THE \#2 STATISTICS UNDER SAMPLE \#2.

THE T TEST ASSUMES ONLY THAT THE TWO SAMPLES ARE

NORMALLY DISTRIBUTED. WE DO NOT ASSUME THAT THE

VARIANCES ARE EQUAL. THIS TEST IS MORE GENERAL

THAN THE USUAL TEST WHICH ASSUMES EQUAL VARIANCES. 
Ver:4 Jul 88

Rapid Data

San Jose CA

DESCRIPTION:
MEAN AND VARIANCE TEST

$50 \mathrm{~nm}$ versus $100 \mathrm{~nm}$ DC Magnetron Carbon

Test for difference in moisture adsorption at 70\% RH

\begin{tabular}{|lrr|l}
\cline { 2 - 3 } \multicolumn{1}{c|}{} & 100 . mm thick & 50 mm thick & \\
\hline DATA POINTS & 4.00 & 7.00 & ENTER THE NUMBR OF DATA POINTS \\
MEAN (ug/cm^^2) & 0.43 & 0.24 & ENTER THE MEAN \\
STD DEV $(\mathrm{N})$ & 0.060 & 0.060 & ENTER THE STANDARD DEVIATION \\
\hline
\end{tabular}

$$
\begin{aligned}
& \mathrm{T}= \\
& \mathrm{DF}= \\
& \mathrm{PROB}(-\mathrm{INF}, \mathrm{T})= \\
& \mathrm{PROB}(-\mathrm{T}, \mathrm{T})= \\
& \mathrm{F}= \\
& \mathrm{DF}(\mathrm{N} 1, \mathrm{~N} 2)= \\
& \operatorname{PROB}(0, \mathrm{~F})=
\end{aligned}
$$

INSTRUCTIONS

1)

2)
4.48 T TEST FOR MEANS. IF $T<1$, THEN USE A TABLE

6.00 DEGREES OF FREEDOM

1.00 PROB OF A SIG DIF IN MEANS (ONE TAIL TEST)

1.00 PROB OF A SIG DIF IN MEANS (TWO TAIL TEST)

1.00 F TEST FOR VARIANCES

6.00 3.00 DEGREES OF FREEDOM

0.54 PROB OF A SIG DIF IN VARIANCES
ENTER THE DESCRIPTION IN THE CORRECT ORDER. FOR EXAMPLE: PROCESS, \#1 vs \#2.

THEN THE \#1 STATISTICS GO UNDER SAMPLE \#1 AND THE \#2 STATISTICS UNDER SAMPLE \#2.

THE T TEST ASSUMES ONLY THAT THE TWO SAMPLES ARE NORMALLY DISTRIBUTED. WE DO NOT ASSUME THAT THE VARIANCES ARE EQUAL. THIS TEST IS MORE GENERAL THAN THE USUAL TEST WHICH ASSUMES EQUAL VARIANCES. 
Ver:4 Jul 88

Rapid Data

San Jose CA

DESCRIPTION:

\begin{tabular}{|lrr|l|}
\cline { 2 - 3 } \multicolumn{1}{c|}{} & Gold & $50 \mathrm{~nm}$ thirk \\
\hline DATA POINTS & 22.00 & 4.00 & ENTER THE NUMBR OF DATA POINTS \\
MEAN (ug/cm^^2) & 0.09 & 0.23 & ENTER THE MEAN \\
STD DEV (N) & 0.016 & 0.060 & ENTER THE STANDARD DEVIATION \\
\hline
\end{tabular}

$\mathrm{T}=$

$\mathrm{DF}=$

PROB $(-I N F, T)=$

$\operatorname{PROB}(-\mathrm{T}, \mathrm{T})=$

$\mathrm{F}=$

$\mathrm{DF}(\mathrm{N} 1, \mathrm{~N} 2)=$

$\operatorname{PROB}(0, F)=$

INSTRUCTIONS

2)
MEAN AND VARIANCE TEST

Gold versus $50 \mathrm{~nm}$ PECVD Carbon

Test for difference in moisture adsorption at 70\% RH

SAMPLES

4.02 T TEST FOR MEANS. IF T<1, THEN USE A TABLE

3.00 DEGREES OF FREEDOM

0.98 PROB OF A SIG DIF IN MEANS (ONE TAIL TEST)

0.97 PROB OF A SIG DIF IN MEANS (TWO TAIL TEST)

14.06 F TEST FOR VARIANCES

3.00 21.00 DEGREES OF FREEDOM

1.00 PROB OF A SIG DIF IN VARIANCES
1)

ENTER THE DESCRIPTION IN THE CORRECT ORDER.

FOR EXAMPLE: PROCESS, \#1 vs \#2.

THEN THE \# I STATISTICS GO UNDER SAMPLE \#1

AND THE \#2 STATISTICS UNDER SAMPLE \#2.

THE T TEST ASSUMES ONLY THAT THE TWO SAMPLES ARE

NORMALLY DISTRIBUTED. WE DO NOT ASSUME THAT THE

VARIANCES ARE EQUAL. THIS TEST IS MORE GENERAL

THAN THE USUAL TEST WHICH ASSUMES EQUAL VARIANCES. 
Ver:4 Jul 88

Rapid Data

San Jose CA

\begin{tabular}{|lrr|r|}
\cline { 2 - 3 } \multicolumn{1}{c|}{} & 100 nm thick & 50 nm thick & \\
\cline { 2 - 3 } \multicolumn{1}{c|}{ DATA POINTS } & 4.00 & 4.00 & ENTER THE NUMBR OF DATA POINTS \\
MEAN (ug/cm^2) & 0.47 & 0.23 & ENTER THE MEAN \\
STD DEV $(\mathrm{N})$ & 0.060 & 0.060 & ENTER THE STANDARD DEVIATION \\
\hline
\end{tabular}

$T=$

$\mathrm{DF}=$

PROB $(-I N F, T)=$

$\operatorname{PROB}(-\mathrm{T}, \mathrm{T})=$

$\mathrm{F}=$

$\mathrm{DF}(\mathrm{N} 1, \mathrm{~N} 2)=$

$\operatorname{PROB}(0, F)=$

INSTRUCTIONS

1)

2)
)
MEAN AND VARIANCE TEST

$50 \mathrm{~nm}$ versus $100 \mathrm{~nm}$ PECVD Carbon

Test for difference in moisture adsorption at $70 \% \mathrm{RH}$

SAMPLES

4.90 T TEST FOR MEANS. IF $T<1$, THEN USE A TABLE

6.00 DEGREES OF FREEDOM

1.00 PROB OF A SIG DIF IN MEANS (ONE TAIL TEST)

1.00 PROB OF A SIG DIF IN MEANS (TWO TAIL TEST)

1.00 F TEST FOR VARIANCES

$3.00 \quad 3.00$ DEGREES OF FREEDOM

0.50 PROB OF A SIG DIF IN VARIANCES
ENTER THE DESCRIPTION IN THE CORRECT ORDER.

FOR EXAMPLE: PROCESS, \#1 vS \#2.

THEN THE \#1 STATISTICS GO UNDER SAMPLE \#1 AND THE \#2 STATISTICS UNDER SAMPLE \#2.

THE T TEST ASSUMES ONLY THAT THE TWO SAMPLES ARE NORMALLY DISTRIBUTED. WE DO NOT ASSUME THAT THE

VARIANCES ARE EQUAL. THIS TEST IS MORE GENERAL

THAN THE USUAL TEST WHICH ASSUMES EQUAL VARIANCES. 
Rapid Data

San Jose CA

DESCRIPTION:

\begin{tabular}{lrr|}
\cline { 2 - 3 } & \multicolumn{1}{c}{ PECVD } & \multicolumn{1}{c|}{ DC } \\
\hline DATA POINTS & 4.00 & 7.00 \\
MEAN (ug/cm^2) & 0.23 & 0.24 \\
STD DEV $(\mathrm{N})$ & 0.060 & 0.060 \\
\hline
\end{tabular}

$\mathrm{T}=$

$\mathrm{DF}=$

PROB(-INF,T) = $\operatorname{PROB}(-\mathrm{T}, \mathrm{T})=$

$F=$

$\mathrm{DF}(\mathrm{N} 1, \mathrm{~N} 2)=$ $\operatorname{PROB}(0, \mathrm{~F})=$

INSTRUCTIONS

1)

2)

FOR EXAMPLE: PROCESS, \#1 vS \#2.
MEAN AND VARIANCE TEST $50 \mathrm{~nm}$ PECVD versus $50 \mathrm{~nm}$ DC Magnetron Carbon Test for difference in moisture adsorption at $70 \% \mathrm{RH}$

\section{SAMPLES}

$0.24 T$ TEST FOR MEANS. IF $T<1$, THEN USE A TABLE 6.00 DEGREES OF FREEDOM

\#N/A PROB OF A SIG DIF IN MEANS (ONE TAIL TEST)

\#N/A PROB OF A SIG DIF IN MEANS (TWO TAIL TEST)

1.00 F TEST FOR VARIANCES

6.00 3.00 DEGREES OF FREEDOM

0.54 PROB OF A SIG DIF IN VARIANCES

ENTER THE DESCRIPTION IN THE CORRECT ORDER.

THEN THE \#1 STATISTICS GO UNDER SAMPLE \#1

AND THE \#2 STATISTICS UNDER SAMPLE \#2.

THE T TEST ASSUMES ONLY THAT THE TWO SAMPLES ARE NORMALLY DISTRIBUTED. WE DO NOT ASSUME THAT THE VARIANCES ARE EQUAL. THIS TEST IS MORE GENERAL THAN THE USUAL TEST WHICH ASSUMES EQUAL VARIANCES. 
Rapid Data

San Jose CA
MEAN AND VARIANCE TEST

$100 \mathrm{~nm}$ PECVD versus $100 \mathrm{~nm}$ DC Magnetron Carbon Test for difference in moisture adsorption at $70 \% \mathrm{RH}$

DESCRIPTION: $\quad$ SAMPLES

\begin{tabular}{|lrr|l}
\cline { 2 - 3 } \multicolumn{1}{c|}{} & PECVD & \multicolumn{1}{c}{ DC } \\
\cline { 2 - 2 } \multicolumn{1}{c|}{ DATA POINTS } & 4.00 & 4.00 & ENTER THE NUMBR OF DATA POINTS \\
MEAN $\left(\mathrm{ug} / \mathrm{cm}^{\wedge} 2\right)$ & 0.47 & 0.43 & ENTER THE MEAN \\
STD DEV $(\mathrm{N})$ & 0.060 & 0.060 & ENTER THE STANDARD DEVIATION \\
\hline
\end{tabular}

$\mathrm{T}=$

$\mathrm{DF}=$

PROB $(-I N F, T)=$

$\operatorname{PROB}(-\mathrm{T}, \mathrm{T})=$

$\mathrm{F}=$

$D F(N 1, N 2)=$

$\operatorname{PROB}(0, F)=$
0.82 T TEST FOR MEANS. IF $T<1$, THEN USE A TABLE

6.00 DEGREES OF FREEDOM

\#N/A PROB OF A SIG DIF IN MEANS (ONE TAIL TEST)

\#N/A PROB OF A SIG DIF IN MEANS (TWO TAIL TEST)

1.00 F TEST FOR VARIANCES

$3.00 \quad 3.00$ DEGREES OF FREEDOM

0.50 PROB OF A SIG DIF IN VARIANCES

INSTRUCTIONS

1)

ENTER THE DESCRIPTION IN THE CORRECT ORDER.

FOR EXAMPLE: PROCESS, \#1 vS \#2.

THEN THE \#1 STATISTICS GO UNDER SAMPLE \#1

AND THE \#2 STATISTICS UNDER SAMPLE \#2.

2)

THE T TEST ASSUMES ONLY THAT THE TWO SAMPLES ARE

NORMALLY DISTRIBUTED. WE DO NOT ASSUME THAT THE

VARIANCES ARE EQUAL. THIS TEST IS MORE GENERAL

THAN THE USUAL TEST WHICH ASSUMES EQUAL VARIANCES. 